\title{
New analytic results for the Zernike circle polynomials from a basic result in the Nijboer-Zernike diffraction theory
}

\author{
A. J. E. M. Janssen \\ a.j.e.m.janssen@tue.nl
}

Department EE and EURANDOM, Eindhoven University of Technology, PO Box 513, 5600 MB Eindhoven, The Netherlands

Several quantities related to the Zernike circle polynomials admit an expression, via the basic identity in the diffraction theory of Nijboer and Zernike, as an infinite integral involving the product of two or three Bessel functions. In this paper these integrals are identified and evaluated explicitly for the cases of (a) the expansion coefficients of scaled-and-shifted circle polynomials, (b) the expansion coefficients of the correlation of two circle polynomials, (c) the Fourier coefficients occurring in the cosine representation of the radial part of the circle polynomials. [DOI: 10.2971/jeos.2011.11028]

Keywords: Diffraction, Zernike Polynomals, Scaling, Shifting, Optical Transfer Function

\section{INTRODUCTION}

Zernike circle polynomials are extensively used in the characterization of circular optical imaging systems with nonuniform pupil functions [1]-[8], and, more recently, for the computation of acoustical quantities arising from harmonically excited, baffled-piston radiators with non-uniform velocity profiles [9]-[11]. The circle polynomials were introduced by Zernike [12] in connection with the knife-edge test and his phase-contrast method and they play a fundamental role in Nijboer's thesis [13] (also see Ch. 9 in [1]), on the diffraction theory of aberrations where they were investigated in detail. Nowadays, the circle polynomials find wide-spread application in optical lithography, astronomy, ophthalmology and other fields dealing with diffraction phenomena involving non-uniformities in a circular, finite setting.

The circle polynomials are given for integer $m, n$ with $n-|m|$ even and non-negative by

$$
Z_{n}^{m}(\rho, \vartheta)=R_{n}^{|m|}(\rho) e^{i m \vartheta}, \quad 0 \leq \rho \leq 1,0 \leq \vartheta<2 \pi,
$$

where the radial polynomials $R_{n}^{|m|}$ are given by

$$
R_{n}^{|m|}(\rho)=\rho^{|m|} P_{\frac{n-|m|}{2}}^{(0,|m|)}\left(2 \rho^{2}-1\right),
$$

with $P_{k}^{(\alpha, \beta)}$ the general Jacobi polynomial as in [18], Ch. 22. The circle polynomials are complete and orthogonal on the unit disk

$$
\begin{gathered}
\left\{(\rho \cos \theta, \rho \sin \theta)=(\nu, \mu) \mid 0 \leq \rho^{2}=v^{2}+\mu^{2} \leq 1,\right. \\
0 \leq \theta<2 \pi\},
\end{gathered}
$$

and can therefore be used to expand any pupil function $P(\rho, \theta)$ that is defined on and square integrable over the unit disk. Besides being complete and orthogonal, the circle polynomials possess a particular convenient form, in terms of Jinc functions (see Eq. (5) below), for their Fourier transforms. This result, which we shall call the basic identity of the NijboerZernike theory of optical aberrations (basic NZ-result, for short) was given by Zernike in [12] and applied by Nijboer in [13] to compute the optical point-spread function in and near the best-focus plane for mildly aberrated, circular optical systems with low-to-medium numerical aperture (NA). The Nijboer-Zernike approach to point-spread computation has been extended in recent years to cover the whole focal region for systems with arbitrarily high NA including the state of polarization and birefringence, see [14]-[17] and [4] where a survey is given.

The basic NZ-result has yielded several useful analytic results in a variety of optical applications. In [5], this result is applied to derive expressions for the derivatives of the radial polynomials, with application to atmospheric turbulence. In [19, 22], a formula for the Zernike expansion coefficients of a scaled pupil in terms of the coefficients of the unscaled pupil is derived. This formula is based on a concise expression for scaled radial polynomials in terms of unscaled radial polynomials, see Eq. (8) below, the proof of which is of similar nature as the one given in [5] and heavily depends on the basic NZ-result. Results on pupil scaling find applications in optical lithography, where the NA of the optical system may be decreased deliberately for imaging enhancement of particular structures, and in ophthalmology, where pupil scaling is studied as a natural attribute of the human eye pupil, see [7] and [20].

The basic NZ-result can also be used to express the Radon transform (the integral along a line of arbitrary distance $p$ to the origin and arbitrary angle $\phi$ of the normal with the $v$ axis) of the circle polynomials, see Eq. (9) below. This result is used in [21] to obtain a computation scheme of the DFTtype, with the inherent efficiency and accuracy that come with DFT-algorithms, for the circle polynomials of arbitrarily large degree $n$ and order $m$, see Eq. (10) below. Eq. (10) was derived 
by a different method in [23], while Eq. (9) was presented already in [24] by Cormack in a medical imaging context.

In $[25,26]$, the problem of computing the Zernike expansion coefficients of the optical transfer function (OTF) from the coefficients of the pupil function is tackled using the basic NZresult. This gives rise to integrals involving the product of three Bessel functions for which there are presented recursion relations in [26].

In the present paper, we give analytic solutions to three problems in optics using the basic NZ-result. All these problems have as a common feature that they give rise, via the basic NZ-result, to integrals involving the product of three Bessel functions that can be evaluated in analytic form. These problems / solutions concern

- determining the Zernike expansion of an arbitrary scaled-and-shifted circle polynomial, extending the pure scaling results in $[19,22]$,

- finding the Zernike expansion of the OTF in terms of the expansion coefficients of the pupil function, thereby solving the problem in $[25,26]$ completely analytically,

- finding the Fourier expansion coefficients of the radial polynomials in which the radial variable $\rho, 0 \leq \rho \leq 1$, is replaced by the numerically more convenient variable $\cos x, 0 \leq x \leq \pi / 2$.

In the next section these three problems are described and motivated in more detail. A fourth problem that gives rise, via the basic NZ-result, to integrals of the product of three Bessel functions, is the computation of the spatial impulse responses that occur in baffled-piston acoustic radiation using Zernike expansion of flexible membranes. This problem is discussed in [27] and [28], Sec. 6.

\section{Basic formulas and overview}

The circle polynomials are given for integer $m, n$ with $n-|m|$ even and non-negative by Eq. (1) and (2). It is customary to refer to $n$ as the degree and to $m$ as the azimuthal order of $Z_{n}^{m}$. The circle polynomials form a complete orthogonal system of functions on the disk $0 \leq \rho \leq 1$, with $Z_{n}^{m}(1, \vartheta)=e^{i m \vartheta}$, which implies that

$$
R_{n}^{|m|}(1)=1
$$

and the orthogonality property reads explicitly

$$
\int_{0}^{12 \pi} \int_{0}^{m} Z_{n}^{m}(\rho, \vartheta)\left(\left(Z_{n^{\prime}}^{m^{\prime}}(\rho, \vartheta)\right)^{*} \rho d \rho d \vartheta=\frac{\pi}{n+1} \delta_{m m^{\prime}} \delta_{n n^{\prime}}\right.
$$

with $\delta$ Kronecker's delta. In the sequel it will be convenient to set $R_{n}^{|m|}=Z_{n}^{m} \equiv 0$ for integer values of $m, n$ such that $n-|m|$ is odd or negative.

A crucial property of the circle polynomials for diffraction theory is that their Fourier transform has the particular simple form

$$
\begin{aligned}
& \iint_{v^{2}+\mu^{2} \leq 1} e^{2 \pi i v x+2 \pi i \mu y} Z_{n}^{m}(\rho, \vartheta) d v d \mu \\
= & \iint_{0}^{12 \pi} e^{2 \pi i \rho r \cos (\vartheta-\varphi)} R_{n}^{|m|}(\rho) e^{i m \vartheta} \rho d \rho d \vartheta \\
= & 2 \pi i^{n} \frac{J_{n+1}(2 \pi r)}{2 \pi r} e^{i m \varphi},
\end{aligned}
$$

where we have written $v+i \mu=\rho e^{i \vartheta}$ and $x+i y=r e^{i \varphi}$. Equivalently, in terms of Hankel transforms (of order $m$ ), we have (using $J_{m}(z)=i^{m-|m|} J_{|m|}(z)$ )

$$
\int_{0}^{1} R_{n}^{|m|}(\rho) J_{m}(2 \pi r \rho) \rho d \rho=(-1)^{\frac{n-m}{2}} \frac{J_{n+1}(2 \pi r)}{2 \pi r} .
$$

This is the basic NZ-result as given in [12], Eq. (23) and [13], Eq. (2.20).

By Fourier inversion in Eq. (5), using Hankel transforms of order $m$, it is seen that

$$
R_{n}^{|m|}(\rho)=(-1)^{\frac{n-|m|}{2}} \int_{0}^{\infty} J_{n+1}(u) J_{|m|}(\rho u) d u, \quad 0 \leq \rho<1 .
$$

This result, often attributed to Noll [5], is shown in [12] to follow from the discontinuous Weber-Schafheitlin integral, see [18], 15.4 .6 on p. 561. The integral on the right-hand side of Eq. (7) converges uniformly in any closed set of $\rho \geq 0$ not containing 1, see Appendix A, and its value for $\rho>1$ is 0 . Thus the equality in (7) holds pointwise and not just in an $L^{2}$-sense. Also see [19], Appendix, Sec. A.1.1 for a discussion of the result in Eq. (7).

The results in Eqs. (6), (7) are basic to the proof of a number of results of the circle polynomials and their radial parts. In [5], the result in Eq. (7) was used to derive expressions for the derivative of $R_{n}^{m}$ in terms of $R$-polynomials of azimuthal orders $m \pm 1$ by employing recurrence relations for Bessel functions and their derivatives. In [19]-[22], the two results in Eqs. (6), (7) were combined with recursion properties of the Bessel functions to produce the scaling formula

$$
\begin{aligned}
R_{n^{\prime}}^{m}(\varepsilon \rho) & =\sum_{n}\left(R_{n^{\prime}}^{n}(\varepsilon)-R_{n^{\prime}}^{n+2}(\varepsilon)\right) R_{n}^{m}(\rho) \\
& =\frac{1}{\varepsilon} \sum_{n} \frac{n+1}{n^{\prime}+1}\left(R_{n^{\prime}+1}^{n+1}(\varepsilon)-R_{n^{\prime}-1}^{n+1}(\varepsilon)\right) R_{n}^{m}(\rho)
\end{aligned}
$$

Here $m=0,1, \ldots, n^{\prime}=m, m+2, \ldots$, and the summation is over $n=m, m+2, \ldots, n^{\prime}$ in which we recall the convention that $R_{n^{\prime}}^{n^{\prime}+2}=R_{n^{\prime}-1}^{n^{\prime}+1} \equiv 0$ for the last term in either series. Although Eq. (8) is normally used for $\varepsilon, \rho \in[0,1]$, it should be emphasized that they are valid for all complex values of $\varepsilon$ and $\rho$ by analyticity. The result in Eq. (8) is of interest to both the lithographic community and the ophthalmological community, see [7], [8], [19]-[20].

In [21], the formula

$$
\begin{aligned}
& \mathcal{R}_{n}^{m}(p, \varphi)=\frac{2}{n+1}\left(1-p^{2}\right)^{1 / 2} U_{n}(p) e^{i m \varphi}, \\
& \text { where } 0 \leq p \leq 1, \quad 0 \leq \varphi<2 \pi,
\end{aligned}
$$


for the Radon transform of $Z_{n}^{m}(\rho, \vartheta)$ is used to show that

$$
R_{n}^{m}(\rho)=\frac{1}{N} \sum_{k=0}^{N-1} U_{n}\left(\rho \cos \frac{2 \pi k}{N}\right) \cos \frac{2 \pi m k}{N},
$$

where $0 \leq \rho \leq 1$.

Here $m=0,1, \ldots$ and $N$ is any integer $>n+m$, and $U_{n}$ is the Chebyshev polynomial of degree $n$ and second kind. This formula is interesting since it gives the $R_{n}^{m}(\rho)$ for $m+n<N$ in the form of a discrete cosine transform, also see [23]. The result in Eq. (9) was discovered by Cormack in [24], but a proof can also be based on the result in Eqs. (6), (7) and the connection between Bessel functions and Chebyshev polynomials through the Fourier transform, see [18], 11.4.24-25 on p. 486.

In the present paper a number of new applications of the results in Eqs. (6), (7) are presented. A common feature of the problems we consider is that they all give rise to infinite integrals involving the product of three Bessel functions. In Section 3 we consider the problem of finding the Zernike expansion of scaled-and-shifted circle polynomials. That is, given $a \geq 0, b \geq 0$ with $a+b \leq 1$, we give explicit expressions, involving Jacobi polynomials, for the coefficients $K_{n n^{\prime}}^{m m^{\prime}}(a, b)$ in the expansion

$$
\begin{aligned}
& Z_{n}^{m}\left(a+b \rho^{\prime} e^{i \vartheta^{\prime}}\right)=\sum_{n^{\prime}, m^{\prime}} K_{n n^{\prime}}^{m m^{\prime}}(a, b) Z_{n^{\prime}}^{m^{\prime}}\left(\rho^{\prime} e^{i \vartheta^{\prime}}\right), \\
& \text { where } 0 \leq \rho^{\prime} \leq 1, \quad 0 \leq \vartheta^{\prime}<2 \pi,
\end{aligned}
$$

see Fig. 1 for the relation between the radial and angular variables of the full, centralized pupil and the reduced, shifted pupil.

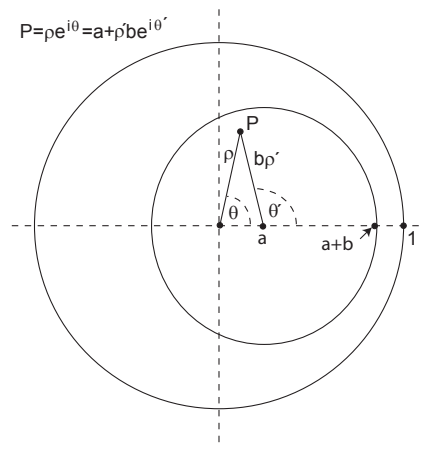

FIG. 1 Centralized, full pupil $\rho e^{i \vartheta}, 0 \leq \rho \leq 1,0 \leq \vartheta<2 \pi$, and shifted and reduced pupil $a+b \rho^{\prime} e^{i \vartheta^{\prime}}, 0 \leq \rho^{\prime} \leq 1,0 \leq \vartheta^{\prime}<2 \pi$, where $a \geq 0, b \geq 0, a+b \leq 1$.

This result generalizes the scaling formulas in Eq. (8) which is the case with $a=0$ in Eq. (11). Furthermore, it gives the analytic solution of the transformation problem for the aberration coefficients of an eye pupil when the pupil is scaled and displaced. This problem has a long history in the ophthalmological community, see [7] and [20] for recent work and survey material, but no closed-form solution seems to have been found thus far. Moreover, by expressing the scaled-andshifted polynomials as linear combinations of the orthogonal terms $Z_{n^{\prime}}^{m^{\prime}}$ one has a handle, via the transformation matrix elements $K_{n n^{\prime}}^{m m^{\prime}}(a, b)$, to tackle the important problem of assessing the condition of a finite set of circle polynomials when they are restricted to subdisks of the unit disk.
In Section 4 we consider the problem of computing the Zernike expansion coefficients of the correlation of two circle polynomials. Having these expansion coefficients available is of great interest when calculating transfer functions in optical imaging, see [29], [30] for early work and motivation concerning this problem in computational optics. The complex pupil function of the lens is expanded as a series involving the circle polynomials. The resulting series with the appropriate Zernike coefficients represent the amplitude and phase of the complex pupil function, including wavelength shift and defocusing. Through the analytic expression for the expansion coefficients of the correlation of two circle polynomials, one has direct access to the optical transfer function over the full bandwidth of the imaging system. This mathematical device is very attractive when calculating, for instance, the pattern transfer in high-resolution optical lithography. Hence, when $m, n, m^{\prime}, n^{\prime}$ are integers such that $n-|m|$ and $n^{\prime}-\left|m^{\prime}\right|$ are even and non-negative, and when we denote for two functions $Z, Z^{\prime} \in L^{2}\left(\mathbb{R}^{2}\right)$ the correlation

$$
\begin{aligned}
&\left(Z * *_{\text {corr }} Z^{\prime}\right)(\nu, \mu)=\iint {\left[\left(\nu+v_{1}, \mu+\mu_{1}\right)\right.} \\
&\left.\times\left(Z^{\prime}\right)^{*}\left(\nu_{1}, \mu_{1}\right)\right] d v_{1} d \mu_{1},
\end{aligned}
$$

we are interested in finding the numbers $\Gamma_{n n^{\prime} n^{\prime \prime}}^{m m^{\prime} m^{\prime \prime}}$ such that

$$
\begin{aligned}
& \frac{(n+1)\left(n^{\prime}+1\right)}{\pi^{2}}\left(Z_{n}^{m} * *_{\text {corr }} Z_{n^{\prime}}^{m^{\prime}}\right)(\rho, \vartheta) \\
& =\sum_{n^{\prime \prime}, m^{\prime \prime}} \frac{n^{\prime \prime}+1}{4 \pi} \Gamma_{n n^{\prime} n^{\prime \prime}}^{m m^{\prime} m^{\prime \prime}} Z_{n^{\prime \prime}}^{m^{\prime \prime}}\left(\frac{1}{2} \rho, \vartheta\right) .
\end{aligned}
$$

Note that $Z_{n}^{m} * *{ }_{c o r r} Z_{n^{\prime}}^{m^{\prime}}$ is supported by the disk around 0 of radius 2 and this requires $Z_{n^{\prime \prime}}^{m^{\prime \prime}}\left(\frac{1}{2} \rho, \vartheta\right)$ at the right-hand side of Eq. (13). This problem has been considered in the optical context by Kintner and Sillitto in [25], [26] in the interest of computing the optical transfer function (OTF) from the Zernike expansion of the pupil function. In [25], [26] a number of results is obtained for the quantities $\Gamma$, but no closed-form solution is given as we obtain here. Moreover, the values of the left-hand side of Eq. (13) are expressed here as integrals involving the product of three Bessel functions.

In optical simulations and/or experiments, a judicious choice of sampling points is important. It now turns out that replacing the radial variable $\rho, 0 \leq \rho \leq 1$, by $\cos x, 0 \leq x \leq \pi / 2$, has the effect that the variation of the radial polynomials $R_{n}^{|m|}$ is spread out uniformly over the $x$-range (see Figure 5 in Section 7). This suggests an adequate pupil sampling strategy. In [31], Subsec. 2.4, a matching procedure, using a separable set of sampling points $\left(\rho_{k}, \vartheta_{l}\right)$ on the disk, for estimating the Zernike expansion coefficients of a pupil from its values at the sampling points is proposed. It turns out that choosing the radial sampling points as $\cos x$, with uniformly spaced $x$ between 0 and $\pi / 2$, produces near-optimal results, in the sense that the resulting method competes with Gaussian quadrature for all relevant azimuthal orders $m$ simultaneously. The fact that the variation of $R_{n}^{|m|}$ is spread uniformly over the $x$-range suggests, furthermore, to apply this substitution when integrals, involving the product of a radial polynomial and a function obtained from the pupil function after azimuthal integration, have to be computed. All this motivates consideration in 
Section 5 of the third problem: Finding the Fourier coefficients of the radial polynomials $R_{n}^{|m|}$ in their cosine-representation. Here we aim at finding the Fourier coefficients $a_{n k}^{m}$ in the representation

$$
R_{n}^{|m|}(\cos x)=\sum_{j=0}^{\lfloor n / 2\rfloor} a_{n, n-2 j}^{m} \cos (n-2 j) x .
$$

The $a_{n k}^{m}$ will be found explicitly, and from this result it is seen that they are all non-negative. It then follows from Eq. (3) that

$$
\left|R_{n}^{|m|}(\rho)\right| \leq \sum_{j=0}^{\lfloor n / 2\rfloor} a_{n, n-2 j}^{m}=R_{n}^{|m|}(1)=1, \quad 0 \leq \rho \leq 1
$$

That $\left|R_{n}^{|m|}(\rho)\right| \leq 1,0 \leq \rho \leq 1$, was proved by Szegö, see [32], 7.2.1 on p. 164 and the references given there, and the nonnegativity of the $a_{n k}^{m}$ was established earlier by Koornwinder [33], Corollary 6.2 on p. 113, as was communicated to the author by Erik Koelink [34]. The explicit form of the $a_{n k}^{m}$ does not seem to have been noted before. With the explicit result for the $a_{n k}^{m}$ available, the computation of the above mentioned integrals can be done directly and very explicitly by choosing the appropriate sampling points, of the form $\cos x$ with equidistant $x$, and using, for instance, DCT-techniques. The fact that the $a_{n k}^{m}$, being all non-negative with sum over $k$ equal to 1 , are all small, renders this approach intrinsic stability.

A word about the notation. We identify complex numbers $z$ with their polar representation $\rho e^{i \vartheta}$ or their Cartesian representation $v+i \mu$, whatever is most convenient in a particular setting. Thus we write things like

$$
Z_{n}^{m}\left(a+\rho^{\prime} b e^{i \vartheta^{\prime}}\right), \quad Z_{n}^{m}(\nu, \mu)
$$

to denote $Z_{n}^{m}(\rho, \vartheta)=R_{n}^{|m|}(\rho) e^{i m \vartheta}$ in which

$$
a+\rho^{\prime} b e^{i \vartheta^{\prime}}=v+i \mu=z=\rho e^{i \vartheta}
$$

with $a, b \geq 0, a+b \leq 1 ; 0 \leq \rho^{\prime} \leq 1,0 \leq \vartheta^{\prime}<2 \pi ; v, \mu \in \mathbb{R}$, $v^{2}+\mu^{2} \leq 1 ; 0 \leq \rho \leq 1,0 \leq \vartheta<2 \pi$.

\section{Scaled-and-shifted Zernike circle polynomials}

We shall prove the following result.

Theorem 3.1. Let $a \geq 0, b \geq 0$ with $a+b \leq 1$, and let $n, m$ be integers with $n-|m|$ even and non-negative. Then

$$
\begin{array}{r}
Z_{n}^{m}\left(a+b \rho^{\prime} e^{i \vartheta^{\prime}}\right)=\sum_{n^{\prime}, m^{\prime}} K_{n n^{\prime}}^{m m^{\prime}}(a, b) Z_{n^{\prime}}^{m^{\prime}}\left(\rho^{\prime} e^{i \vartheta^{\prime}}\right), \\
\text { where } 0 \leq \rho^{\prime} \leq 1,0 \leq \vartheta^{\prime} \leq 2 \pi,
\end{array}
$$

where for $n=|m|,|m|+2, \ldots, n^{\prime}=\left|m^{\prime}\right|,\left|m^{\prime}\right|+2, \ldots$

$$
K_{n n^{\prime}}^{m m^{\prime}}(a, b)=T_{n n^{\prime}}^{m m^{\prime}}(a, b)-T_{n, n^{\prime}+2}^{m m^{\prime}}(a, b) .
$$

Here

$$
T_{n n^{\prime \prime}}^{m m^{\prime \prime}}=(-1)^{p-p^{\prime \prime}} \int_{0}^{\infty} J_{m-m^{\prime \prime}}(a u) J_{n^{\prime \prime}}(b u) J_{n+1}(u) d u .
$$

In the case when $n-n^{\prime \prime} \geq m-m^{\prime \prime} \geq 0$, we have

$$
\begin{aligned}
T_{n n^{\prime \prime}}^{m m^{\prime \prime}}= & \frac{\left(q+p^{\prime \prime}\right) !\left(p-p^{\prime \prime}\right) !}{\left(q-q^{\prime \prime}\right) !\left(p+q^{\prime \prime}\right) !} a^{m-m^{\prime \prime}} b^{n^{\prime \prime}} \\
& \times P_{p-p^{\prime \prime}}^{\left(m-m^{\prime \prime}, n^{\prime \prime}\right)}\left(1-2 A^{2}\right) P_{p-p^{\prime \prime}}^{\left(m-m^{\prime \prime}, n^{\prime \prime}\right)}\left(2 B^{2}-1\right) .
\end{aligned}
$$

In the case when $n-n^{\prime \prime} \geq m^{\prime \prime}-m \geq 0$, we have

$$
\begin{aligned}
T_{n n^{\prime \prime}}^{m m^{\prime \prime}}= & \frac{\left(p+q^{\prime \prime}\right) !\left(q-q^{\prime \prime}\right) !}{\left(p-p^{\prime \prime}\right) !\left(q+p^{\prime \prime}\right) !} a^{m^{\prime \prime}-m} b^{n^{\prime \prime}} \\
& \times P_{q-q^{\prime \prime}}^{\left(m^{\prime \prime}-m, n^{\prime \prime}\right)}\left(1-2 A^{2}\right) P_{q-q^{\prime \prime}}^{\left(m^{\prime \prime}-m, n^{\prime \prime}\right)}\left(2 B^{2}-1\right) .
\end{aligned}
$$

In any other case, we have

$$
T_{n n^{\prime \prime}}^{m m^{\prime \prime}}=0 .
$$

In Eqs. (20)-(22) we have written

$$
\begin{aligned}
p & =\frac{n-m}{2}, q=\frac{n+m}{2}, \\
p^{\prime \prime} & =\frac{n^{\prime \prime}-m^{\prime \prime}}{2}, q^{\prime \prime}=\frac{n^{\prime \prime}+m^{\prime \prime}}{2} .
\end{aligned}
$$

Furthermore, $P_{k}^{(\gamma, \delta)}(x)$ is the general Jacobi polynomial as in [18], Ch. 22 of degree $k=0,1, \ldots$ corresponding to the weight function $(1-x)^{\gamma}(1+x)^{\delta}$ on the interval $[-1,1]$. Finally,

$$
\begin{aligned}
1-2 A^{2}= & {\left[\left(1-(a+b)^{2}\right)\left(1-(a-b)^{2}\right)\right]^{1 / 2} } \\
& -(a+b)(a-b), \\
2 B^{2}-1= & -\left[\left(1-(a+b)^{2}\right)\left(1-(a-b)^{2}\right)\right]^{1 / 2} \\
& -(a+b)(a-b) .
\end{aligned}
$$

Alternatively, we have

$$
A=\sin \alpha, \quad B=\sin \beta
$$

with $\alpha \geq 0, \beta \geq 0$ such that $\alpha+\beta \leq \pi / 2$ and

$$
a=\sin \alpha \cos \beta, \quad b=\cos \alpha \sin \beta .
$$

That is, $A$ and $B$ can be obtained from the geometrical picture in Figure 2 where $\gamma \in\left[\frac{\pi}{2}, \pi\right]$ is such that $\sin \gamma=a+b$.

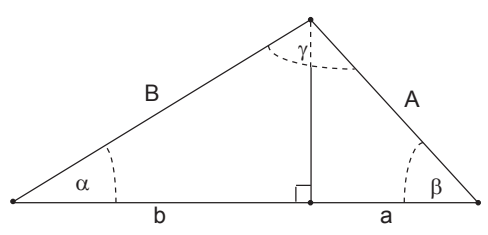

FIG. 2 Geometric definition of angles $\alpha, \beta, \gamma$ and side lengths $A, B$ from $a \geq 0, b \geq 0$, $a+b \leq 1$ in accordance with Eqs. (27), (28) and the rule of sines $\sin \alpha / A=\sin \beta / B=\sin \gamma /(a+b)=1$.

Proof. By completeness and orthogonality of the circle polynomials, see Eq. (4), we have that

$$
\begin{aligned}
& K_{n n^{\prime}}^{m m^{\prime}}(a, b)=\frac{n^{\prime}+1}{\pi} \int_{0}^{1} \int_{0}^{2 \pi}[ Z_{n}^{m}\left(a+b \rho^{\prime} e^{i \vartheta^{\prime}}\right) \\
&\left.\times\left(Z_{n^{\prime}}^{m^{\prime}}\left(\rho^{\prime} e^{i \vartheta^{\prime}}\right)\right)^{*} \rho^{\prime} d \rho^{\prime} d \vartheta^{\prime}\right] .
\end{aligned}
$$


We write $\rho e^{i \vartheta}=a+\rho^{\prime} b e^{i \vartheta^{\prime}}$ in which $\rho$ and $\vartheta$ are depending on $\rho^{\prime}$, $\vartheta^{\prime}$ with $0 \leq \rho^{\prime} \leq 1,0 \leq \vartheta^{\prime} \leq 2 \pi$. Then we get

$$
\begin{aligned}
K_{n n^{\prime}}^{m m^{\prime}}(a, b)= & \frac{n^{\prime}+1}{\pi} \int_{0}^{1} \int_{0}^{2 \pi}\left[R_{n}^{|m|}\left(\rho\left(\rho^{\prime}, \vartheta^{\prime}\right)\right)\right. \\
& \left.\times e^{i m \vartheta\left(\rho^{\prime}, \vartheta^{\prime}\right)} R_{n^{\prime}}^{\left|m^{\prime}\right|}\left(\rho^{\prime}\right) e^{-i m^{\prime} \vartheta^{\prime}} \rho^{\prime} d \rho^{\prime} d \vartheta^{\prime}\right] .
\end{aligned}
$$

We now use Eq. (7) to rewrite $R_{n}^{|m|}\left(\rho\left(\rho^{\prime}, \vartheta^{\prime}\right)\right)$ in integral form and change the order of integration; this is allowed on account of Appendix A where we show that the integral on the righthand side of Eq. (7) converges boundedly for all $\rho \geq 0$ and uniformly, to $(-1)^{(n-|m|) / 2} R_{n}^{|m|}(\rho)$, on any set $[0,1-\varepsilon]$ with $\varepsilon>0$. Therefore,

$$
\begin{aligned}
& K_{n n^{\prime \prime}}^{m m^{\prime}}(a, b)=\frac{n^{\prime}+1}{\pi}(-1)^{\frac{n-|m|}{2}} \int_{0}^{\infty} J_{n+1}(u) \\
& \times\left[\int_{0}^{1}\left\{\int_{0}^{2 \pi} J_{|m|}\left(u \rho\left(\rho^{\prime}, \vartheta^{\prime}\right)\right) e^{i m \vartheta\left(\rho^{\prime}, \vartheta^{\prime}\right)} e^{-i m^{\prime} \vartheta^{\prime}} d \vartheta^{\prime}\right\}\right. \\
& \left.\quad \times R_{m}^{\left|m^{\prime}\right|}\left(\rho^{\prime}\right) \rho^{\prime} d \rho^{\prime}\right] d u .
\end{aligned}
$$

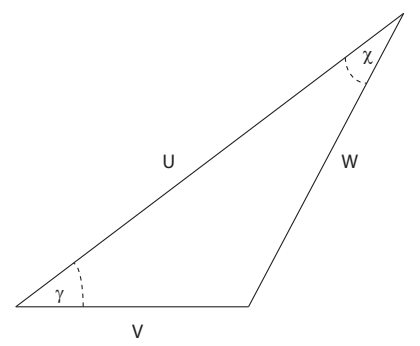

FIC. 3 Geometric relation between angles $\chi, \gamma$ and side lengths $U, V, W$ for Eq. (32).

We use the addition theorem of Graf, see [18], 9.1.79 on p. 363,

$$
\mathcal{C}_{l}(W) e^{i l \chi}=\sum_{k=-\infty}^{\infty} \mathcal{C}_{l+k}(U) J_{k}(V) e^{i k \gamma}
$$

for integer $l$ and $\mathcal{C}=J$, where $W, U, V, \chi$ and $\gamma$ are related as in the picture in Figure 3. With the variables $\rho^{\prime}, \vartheta^{\prime}, \rho, \vartheta$ as in the integral in Eq. (31) and displayed in Figure 1, we use Eq. (32) with

$$
\begin{array}{r}
W=u \rho\left(\rho^{\prime}, \vartheta^{\prime}\right), \quad U=u a, \quad V=u \rho^{\prime} b, \\
\chi=\vartheta\left(\rho^{\prime}, \vartheta^{\prime}\right), \quad \gamma=\pi-\vartheta^{\prime},
\end{array}
$$

where we note by the comments in [18] after 9.1.79 and 9.1.80 on p. 363 that Eq. (32) can be used without any further restriction on $U, V, W$. Then we get

$$
\begin{aligned}
& J_{m}\left(u \rho\left(\rho^{\prime}, \vartheta^{\prime}\right)\right) e^{i m \vartheta\left(\rho^{\prime}, \vartheta^{\prime}\right)} \\
& =\sum_{k=-\infty}^{\infty} J_{m+k}(u a) J_{k}\left(u \rho^{\prime} b\right) e^{i k\left(\pi-\vartheta^{\prime}\right)} .
\end{aligned}
$$

Hence, in the case that $m \geq 0$,

$$
\begin{aligned}
& \int_{0}^{2 \pi} J_{m}\left(u \rho\left(\rho^{\prime}, \vartheta^{\prime}\right)\right) e^{i m \vartheta\left(\rho^{\prime}, \vartheta^{\prime}\right)} e^{-i m^{\prime} \vartheta^{\prime}} d \vartheta^{\prime} \\
& =2 \pi(-1)^{m^{\prime}} J_{m-m^{\prime}}(u a) J_{-m^{\prime}}\left(u \rho^{\prime} b\right) \\
& =2 \pi J_{m-m^{\prime}}(u a) J_{m^{\prime}}(u \rho b),
\end{aligned}
$$

and so

$$
\begin{aligned}
K_{n n^{\prime}}^{m m^{\prime}}(a, b)= & 2\left(n^{\prime}+1\right)(-1)^{\frac{n-m}{2}} \int_{0}^{\infty}\left\{J_{n+1}(u) J_{m-m^{\prime}}(u a)\right. \\
& \left.\times\left[\int_{0}^{1} J_{m^{\prime}}\left(u \rho^{\prime} b\right) R_{n^{\prime}}^{\left|m^{\prime}\right|}\left(\rho^{\prime}\right) \rho^{\prime} d \rho^{\prime}\right] d u\right\} .
\end{aligned}
$$

Using Eq. (34) for $m<0$ and noting that

$J_{|m|}(z)=(-1)^{m} J_{m}(z)$ while

$$
(-1)^{\frac{n-|m|}{2}}(-1)^{m}=(-1)^{\frac{n-m}{2}}
$$

for $m<0$, it is seen that Eq. (36) holds for all integer $m$.

Next we use Eq. (6) with $m^{\prime}, n^{\prime}, u b$ instead of $m, n, 2 \pi r$ to rewrite the integral in [ ] in Eq. (36), and we obtain

$$
\begin{aligned}
K_{n n^{\prime}}^{m m^{\prime}}(a, b)= & 2\left(n^{\prime}+1\right)(-1)^{\frac{n+n^{\prime}-m-m^{\prime}}{2}} \\
& \times \int_{0}^{\infty} \frac{J_{n+1}(u) J_{m-m^{\prime}}(u a) J_{n^{\prime}+1}(u b)}{u b} d u .
\end{aligned}
$$

Using [18], first item in 9.1.27 on p. 361,

$$
\frac{J_{n^{\prime}+1}(z)}{z}=\frac{1}{2\left(n^{\prime}+1\right)}\left(J_{n^{\prime}}(z)+J_{n^{\prime}+2}(z)\right),
$$

it then follows that

$$
\begin{aligned}
K_{n n^{\prime}}^{m m^{\prime}}(a, b)= & (-1)^{\frac{n+n^{\prime}-m-m^{\prime}}{2}} \\
& \times\left[\int_{0}^{\infty} J_{n+1}(u) J_{m-m^{\prime}}(u a) J_{n^{\prime}}(u b) d u\right. \\
& \left.+\int_{0}^{\infty} J_{n+1}(u) J_{m-m^{\prime}}(u a) J_{n^{\prime}+2}(u b) d u\right],
\end{aligned}
$$

and this establishes the equality in Eq. (19) with T's given in integral form in Eq. (20). The identities in Eq. (21)-(23) follow from an application of a result of Bailey, the administrative details of which are deferred to Section 6.

We now list some special cases of Theorem 3.1.

- $a=0$. This gives the result of the scaling theory as developed in [19], [22], also see Eq. (8). To see this, note that in Eqs.(27), (28) we have

$$
A=a=\alpha=0, \quad B=b=\sin \beta,
$$

and in Eqs. (19),(20) only the cases with $m=m^{\prime}$ give non-zero results. Furthermore,

$$
P_{k}^{\left(0, n^{\prime}\right)}(1)=1, \quad b^{n^{\prime}} P_{k}^{\left(0, n^{\prime}\right)}\left(2 b^{2}-1\right)=R_{n^{\prime}+2 k}^{n^{\prime}}(b) ，
$$

and, see Eq. (24),

$$
p+q^{\prime}=p^{\prime}+q, \quad p-p^{\prime}=q-q^{\prime}
$$

since $m=m^{\prime}$. Plugging all this in into Eq. (21) yields Eq. (8).

$-b=0$. Only $n^{\prime}=0$ gives non-zero results in Eqs 
and then also $m^{\prime}=0$ (since $\left.\left|m^{\prime}\right| \leq n^{\prime}\right)$. Now we have in Eqs.(27), (28)

$$
A=a=\sin \alpha, \quad B=b=\beta=0,
$$

and, see Eq. (24),

$$
q+p^{\prime}=q-q^{\prime}, \quad p-p^{\prime}=p+q^{\prime}
$$

since $p^{\prime}=q^{\prime}=m^{\prime}=n^{\prime}=0$. Thus, when $m \geq 0$, Eq. (21) yields

$$
\begin{aligned}
& a^{m} P_{p}^{(m, 0)}\left(1-2 a^{2}\right) P_{p}^{(m, 0)}(-1) \\
& =a^{m} P_{p}^{(0, m)}\left(2 a^{2}-1\right) \\
& =R_{m+2 p}^{m}(a)=R_{n}^{m}(a)
\end{aligned}
$$

where we have used that

$$
P_{k}^{(\gamma, \delta)}(-x)=(-1)^{k} P_{k}^{(\delta, \gamma)}(x), \quad P_{p}^{(0, m)}(1)=1 .
$$

Therefore, we have in Eq. (18) with $b=0$ the trivial representation

$$
Z_{n}^{m}(a)=R_{n}^{m}(a) Z_{0}^{0}\left(\rho^{\prime}\right), \quad 0 \leq \rho^{\prime} \leq 1,0 \leq \vartheta^{\prime} \leq 2 \pi,
$$

with a similar result in the case that $m \leq 0$.

$-a+b=1$. We have in Eq. (27),(28) in this limit case

$$
\begin{gathered}
\alpha+\beta=\pi / 2, A=\sin \alpha, B=\cos \alpha, \\
1-2 A^{2}=2 B^{2}-1=\cos 2 \alpha .
\end{gathered}
$$

As a consequence, all T's are non-negative.

A further interesting observation is that

$$
K_{n n^{\prime}}^{m m^{\prime}}(a, b) \neq 0 \Rightarrow\left|m^{\prime}\right| \leq n^{\prime} \leq n-\left|m-m^{\prime}\right| .
$$

Hence, a Zernike circle polynomials $Z_{n}^{m}$ can be identified from the set of integer pairs $\left(m^{\prime}, n^{\prime}\right)$ corresponding to non-zero coefficients when $Z_{n}^{m}$ is scaled and shifted. In Subsection 7.1, a detailed computation based on Theorem 3.1 of the Zernike expansion of the scaled-and-shifted circle polynomial $Z_{4}^{0}$ is presented.

The validity of the identities (21)-(23) for $T$ in Theorem 3.1 will now be shown to extend to all complex values of $a$ and $b$. First assume that $a \geq 0, b \geq 0, a+b \leq 1$. We have from Eqs. (1), (2) that

$$
Z_{n}^{m}\left(\rho e^{i \vartheta}\right)=\left(\rho e^{ \pm i \vartheta}\right)^{|m|} P_{\frac{n-|m|}{2}}^{(0,|m|)}\left(2 \rho^{2}-1\right),
$$

where $\pm=\operatorname{sgn}(m)$. Since

$$
\begin{aligned}
\rho e^{ \pm i \vartheta} & =a+b \rho^{\prime} e^{ \pm i \vartheta^{\prime}}, \\
\rho^{2} & =a^{2}+b^{2}\left(\rho^{\prime}\right)^{2}+2 a b \rho^{\prime} \cos \vartheta^{\prime},
\end{aligned}
$$

we have that

$$
Z_{n}^{m}\left(a+b \rho^{\prime} e^{i \vartheta^{\prime}}\right)=\sum_{k=-\frac{n-|m|}{2}}^{\frac{n+|m|}{2}} p_{k}\left(\rho^{\prime} ; a, b\right) e^{ \pm i k \vartheta^{\prime}},
$$

where $p_{k}\left(\rho^{\prime} ; a, b\right)$ depends polynomially on $\rho^{\prime}, a$ and $b$. On the other hand, from Eqs. (25), (26), we have that for any polynomial $p$

$$
p\left(1-2 A^{2}\right) p\left(2 B^{2}-1\right)=p(-x+y) p(-x-y)
$$

is an even function of $y=\left(\left(1-(a+b)^{2}\right)\left(1-(a-b)^{2}\right)\right)^{1 / 2}$ for any value of $x=(a+b)(a-b)$. Consequently, the right-hand side of Eq. (54) contains only even powers of $y$. We conclude that any of the T's considered in Eqs. (21)-(23) depends polynomially on $a$ and $b$. Hence, the relation in Eq. (18) extends to all $a, b \in \mathbb{C}$ by analyticity in which $Z_{n}^{m}\left(a+b \rho^{\prime} e^{i \vartheta^{\prime}}\right)$ at the righthand side of Eq. (18) is to be replaced by the analytic extension $W_{n}^{m}\left(a, b, \rho^{\prime}, \vartheta^{\prime}\right)$ of the right-hand side of Eq. (53) to all complex values of $a, b$ and $\rho^{\prime}$ with $0 \leq \vartheta^{\prime}<2 \pi$. Note that for general complex $a, b, \rho^{\prime}$ and $\vartheta^{\prime}, 0 \leq \vartheta^{\prime}<2 \pi$,

$$
W_{n}^{m}\left(a, b, \rho^{\prime}, \vartheta^{\prime}\right)=Z_{n}^{m}\left(a+b \rho^{\prime} e^{i \vartheta^{\prime}}\right)
$$

does not need to hold even when $\left|a+b \rho^{\prime} e^{i \vartheta^{\prime}}\right| \leq 1$. For instance, when $\alpha$ is real and; $0 \leq \rho^{\prime} \leq 1,0 \leq \vartheta^{\prime}<2 \pi$ with $(a=0, b=\exp (i \alpha)$ in Eq. (55)), we have

$$
W_{n}^{m}\left(0, e^{i \alpha}, \rho^{\prime}, \vartheta^{\prime}\right)=\left(e^{i \alpha} \rho^{\prime} e^{ \pm i \vartheta^{\prime}}\right)^{|m|} R_{n}^{|m|}\left(e^{i \alpha} \rho^{\prime}\right)
$$

while

$$
Z_{n}^{m}\left(e^{i \alpha} \rho^{\prime} e^{i \vartheta^{\prime}}\right)=\left(\rho^{\prime} e^{ \pm i\left(\vartheta^{\prime}+\alpha\right)}\right)^{|m|} R_{n}^{|m|}\left(\rho^{\prime}\right) .
$$

We do have equality in Eq. (55) when $a$ and $b$ are real and $\rho^{\prime} \geq 0,0 \leq \vartheta^{\prime}<2 \pi$, and the definition of $Z_{n}^{m}$ in Eqs. (1), (2) is used with general $\rho \geq 0$. Accordingly, Theorem 3.1 is valid for all real $a$ and $b$, with $Z_{n}^{m}$ defined as in Eqs. (1), (2) with general $\rho \geq 0$.

An important consequence of this extension is that now also the transformation matrices $\left(K_{n n^{\prime}}^{m m^{\prime}}(-a / b, 1 / b)\right)$ corresponding to the inverse transformation $z \mapsto-a / b+z / b$ can be considered. Accordingly, when the degrees $n, n^{\prime}$ are restricted to a finite set $\{0, \ldots, N\}$, the matrices corresponding to $z \mapsto a+b z$ and $z \mapsto-a / b+z / b$ are each other's inverse. Having expanded the scaled-and-shifted circle polynomials in terms of the orthogonal functions $Z_{n^{\prime}}^{m^{\prime}}$, we have now the opportunity to deal with the problem of assessing the condition of the set of circle polynomials of maximal degree $N$ as a linear system when they are restricted to an arbitrary disk in the plane. Indeed, the condition number is given as the square-root of the ratio of the largest and smallest eigenvalue of the Grammian matrix, and this Grammian matrix and its inverse are expressible in terms of the appropriate transformation matrices $\left(K_{n n^{\prime}}^{m m^{\prime}}(a, b)\right)$. Such an effort is already worthwhile for the case of restriction of circle polynomials to a disk $\rho \leq \varepsilon$ with $\varepsilon<1$ (pure scaling), and the author has found for this case useful and simple estimates for the magnitude of these condition numbers. This case is much simpler than the general case since the transformation matrices decouple per $m$, while the issue of analytic extension can be considered on the level of Eq. (8) that extends to all complex $\varepsilon$ and $\rho$.

We finally note that the approach, via the basic NZ-result and Eq. (34), to prove Theorem 3.1 can be used to derive an addition theorem in which the general scaled-and-shifted circle polynomial $Z_{n}^{m}\left(a+b \rho^{\prime} e^{i \theta^{\prime}}\right)$ is expressed as a finite Fourier series in $\theta^{\prime}$ where the Fourier coefficients are (again) in the form of the integral of the product of three Bessel function. This addition theorem generalizes the familiar one for the Legendre polynomials $(m=0)$. 


\section{Zernike expansion of the optical transfer function}

In this section we assume that we have expanded the generalized complex pupil function $P(\rho, \vartheta)$ (vanishing outside $\rho \leq 1$ ) as

$$
\begin{aligned}
& P(\rho, \vartheta)=\sum_{n, m} \frac{n+1}{\pi} \gamma_{n}^{m} Z_{n}^{m}\left(\rho e^{i \vartheta}\right), \\
& \text { where } 0 \leq \rho \leq 1, \quad 0 \leq \vartheta<2 \pi,
\end{aligned}
$$

with coefficients $\gamma_{n}^{m}$ that can be obtained by using orthogonality of the $Z_{n}^{m}$. Writing $v+i \mu=\rho e^{i \vartheta}$ with $v, \mu \in \mathbb{R}$ and identifying $P(\nu, \mu) \equiv P(\rho, \vartheta)$, compare end of Section 2, it is required to find the Zernike expansion of the OTF (optical transfer function)

$$
\begin{aligned}
\left(P * *_{\mathrm{corr}} P\right)(\nu, \mu)=\iint[ & P\left(v+v_{1}, \mu+\mu_{1}\right) \\
\times & \left.P^{*}\left(v_{1}, \mu_{1}\right) d v_{1} d \mu_{1}\right]
\end{aligned}
$$

that vanishes outside the set $v^{2}+\mu^{2} \leq 4$, see Figure 4 . Thus, considering the expansion in Eq. (58), it is required to compute (the Zernike expansion of) $Z_{n}^{m} * *_{\text {corr }} Z_{n^{\prime}}^{m^{\prime}}$ for integer $n, m, n^{\prime}, m^{\prime}$ with $n-|m|$ and $n^{\prime}-\left|m^{\prime}\right|$ even and non-negative. We maintain the $p, q$-notation of Eq. (24).

Theorem 4.1. We have, with $v+i \mu=\rho e^{i \vartheta}$ and $0 \leq \rho \leq 2$,

$$
\begin{aligned}
& \left(Z_{n}^{m} * *_{\operatorname{corr}} Z_{n^{\prime}}^{m^{\prime}}\right)(\nu, \mu)=2 \pi(-1)^{p-p^{\prime}} \\
& \times e^{i\left(m-m^{\prime}\right) \vartheta} \int_{0}^{\infty} \frac{J_{n+1}(u) J_{n^{\prime}+1}(u) J_{m-m^{\prime}}(\rho u)}{u} d u
\end{aligned}
$$

and

$$
\begin{gathered}
\frac{(n+1)\left(n^{\prime}+1\right)}{\pi^{2}}\left(Z_{n}^{m} * *_{\operatorname{corr}} Z_{n^{\prime}}^{m^{\prime}}\right)\left(\rho e^{i \vartheta}\right) \\
=\sum_{n^{\prime \prime}, m^{\prime \prime}} \frac{n^{\prime \prime}+1}{4 \pi} \Gamma_{n n^{\prime} n^{\prime \prime}}^{m m^{\prime} m^{\prime \prime}} Z_{n^{\prime \prime}}^{m^{\prime \prime}}\left(\frac{1}{2} \rho e^{i \vartheta}\right),
\end{gathered}
$$

where $\Gamma$ is non-vanishing for $m^{\prime \prime}=m-m^{\prime}$ only, and in that case

$$
\begin{aligned}
\Gamma_{n n^{\prime} n^{\prime \prime}}^{m m^{\prime} m^{\prime \prime}}= & 8(n+1)\left(n^{\prime}+1\right)(-1)^{\frac{n-n^{\prime}-n^{\prime \prime}}{2}} \\
& \times \int_{0}^{\infty} J_{n+1}(u) J_{n^{\prime}+1}(u) J_{n^{\prime \prime}+1}(2 u) \frac{d u}{u^{2}} .
\end{aligned}
$$

Proof. We have by Parseval's theorem and Eq. (5) that $(\mathcal{F}$ denotes the Fourier transform)

$$
\begin{aligned}
& \left(Z_{n}^{m} * *_{\operatorname{corr}} Z_{n^{\prime}}^{m^{\prime}}\right)(v, \mu) \\
& =\iint \mathcal{F}\left[Z_{n}^{m}(v+\cdot, \mu+\cdot)\right](x, y)\left(\mathcal{F} Z_{n^{\prime}}^{m^{\prime}}\right)^{*}(x, y) d x d y \\
& =\iint e^{-2 \pi i v x-2 \pi i \mu y}\left(\mathcal{F} Z_{n}^{m}\right)(x, y)\left(\mathcal{F} Z_{n^{\prime}}^{m^{\prime}}\right)^{*}(x, y) d x d y \\
& =i^{n-n^{\prime}} \int_{0}^{\infty} \int_{0}^{2 \pi} \frac{J_{n+1}(2 \pi r) J_{n^{\prime}+1}(2 \pi r)}{r^{2}} \\
& \quad \times e^{-2 \pi i \rho r \cos (\vartheta-\varphi)} e^{i\left(m-m^{\prime}\right) \varphi} r d r d \varphi,
\end{aligned}
$$

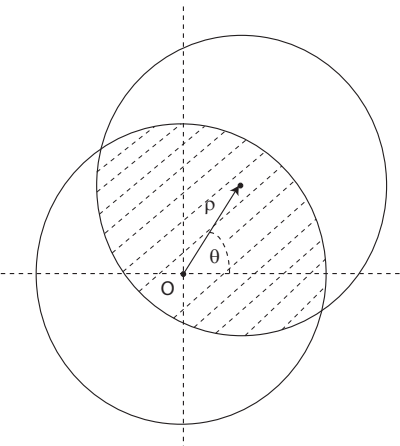

FIG. 4 Schematic representation of the autocorrelation function $P * *_{\operatorname{corr}} P$ of a nonuniform pupil function $P$ as an integral over the common region of two disks of unit radius with centers at 0 and $\rho e^{i \vartheta}$, respectively.

where we have written $x+i y=r e^{i \varphi}$ and where we use that $v x+\mu y=\rho r \cos (\vartheta-\varphi)$. Now

$$
\int_{0}^{2 \pi} e^{-2 \pi i \rho r \cos (\vartheta-\varphi)} e^{i k \varphi} d \varphi=2 \pi(-i)^{k} e^{i k \vartheta} J_{k}(2 \pi \rho r),
$$

and inserting this into Eq. (63) with $k=m-m^{\prime}$, noting that $i^{n-n^{\prime}}(-i)^{m-m^{\prime}}=(-1)^{p-p^{\prime}}$ and substituting $u=2 \pi r$, we obtain Eq. (60).

Next to show Eq. (62), we note from orthogonality of the $Z_{n^{\prime \prime}}^{m^{\prime \prime}}\left(\frac{1}{2} \rho e^{i \vartheta}\right)$, see Eq. (4), that

$$
\begin{aligned}
& \Gamma_{n n^{\prime} n^{\prime \prime}}^{m m^{\prime} m^{\prime \prime}}=\frac{(n+1)\left(n^{\prime}+1\right)}{\pi^{2}} \\
& \times \iint\left(Z_{n}^{m} * *_{\operatorname{corr}} Z_{n^{\prime}}^{m^{\prime}}\right)(\nu, \mu)\left(Z_{n^{\prime \prime}}^{m^{\prime \prime}}\left(\frac{1}{2} \nu, \frac{1}{2} \mu\right)\right)^{*} d \nu d \mu .
\end{aligned}
$$

Again using Parseval's theorem, together with

$$
\begin{aligned}
\mathcal{F}\left(Z * *_{\text {corr }} Z^{\prime}\right) & \left.=\mathcal{F} Z \cdot\left(\mathcal{F} Z^{\prime}\right)^{*}, \quad\left(\mathcal{F} Z^{\prime \prime}\right)\left(\frac{1}{2} v, \frac{1}{2} \mu\right)\right)(x, y) \\
& =4\left(\mathcal{F} Z^{\prime \prime}\right)(2 x, 2 y),
\end{aligned}
$$

we obtain

$$
\begin{aligned}
\Gamma_{n n^{\prime} n^{\prime \prime}}^{m m^{\prime} m^{\prime \prime}}= & \frac{4(n+1)\left(n^{\prime}+1\right)}{\pi^{2}} \iint\left[\left(\mathcal{F} Z_{n}^{m}\right)(x, y)\right. \\
& \left.\times\left(\mathcal{F} Z_{n^{\prime}}^{m^{\prime}}\right)^{*}(x, y)\left(\mathcal{F} Z_{n^{\prime \prime}}^{m^{\prime \prime}}\right)^{*}(2 x, 2 y)\right] d x d y .
\end{aligned}
$$

Then inserting Eq. (5) and using polar coordinates $x+i y=r e^{i \varphi}$, we obtain

$$
\begin{aligned}
\Gamma_{n n^{\prime} n^{\prime \prime}}^{m m^{\prime} m^{\prime \prime}}= & \frac{2(n+1)\left(n^{\prime}+1\right)}{\pi^{2}} i^{n-n^{\prime}-n^{\prime \prime}} \\
& \times \int_{0}^{\infty} J_{n+1}(2 \pi r) J_{n^{\prime}+1}(2 \pi r) J_{n^{\prime \prime}+1}(4 \pi r) \frac{d r}{r^{2}} \\
& \times \int_{0}^{2 \pi} e^{i\left(m-m^{\prime}-m^{\prime \prime}\right) \varphi} d \varphi .
\end{aligned}
$$

The proof is completed by the substitution $u=2 \pi r$. Also note that $n-n^{\prime}$ and $n^{\prime \prime}$ have the same parity when $m^{\prime \prime}=m-m^{\prime}$. 
Notes.

1. We have by Eq. (39) that

$$
\begin{aligned}
\left(Z_{n}^{m} * *_{\text {corr }} Z_{n^{\prime}}^{m^{\prime}}\right)(\nu, \mu)= & \frac{\pi}{n^{\prime}+1}(-1)^{p-p^{\prime}} e^{i\left(m-m^{\prime}\right) \vartheta} \\
& \times\left[Q_{n+1, n^{\prime}}^{m-m^{\prime}}+Q_{n+1, n^{\prime}+2}^{m-m^{\prime}}\right]
\end{aligned}
$$

and, for the case that $m^{\prime \prime}=m-m^{\prime}$,

$$
\begin{aligned}
\Gamma_{n n^{\prime} n^{\prime \prime}}^{m m^{\prime} m^{\prime \prime}}= & 2(-1)^{\frac{n-n^{\prime}-n^{\prime \prime}}{2}} \\
& \times\left[Q_{n n^{\prime}}^{n^{\prime \prime}+1}+Q_{n+2, n^{\prime}}^{n^{\prime \prime}+1}+Q_{n, n^{\prime}+2}^{n^{\prime \prime}+1}+Q_{n+2, n^{\prime}+2}^{n^{\prime \prime}+1}\right],
\end{aligned}
$$

where

$$
Q_{i j}^{k}(a, b, c)=\int_{0}^{\infty} J_{i}(a u) J_{j}(b u) J_{k}(c u) d u,
$$

and where the $Q^{\prime} \mathrm{s}$ in Eq. (69) are evaluated at $(a=1, b=1, c=\rho)$ and the $Q^{\prime}$ s in Eq. (70) are evaluated at $(a=1, b=1, c=2)$.

2. We shall use in Section 6 a result of Bailey [35] to show the following. We have $Q_{n n^{\prime}}^{n^{\prime \prime}+1}=0$ when $n^{\prime \prime}<n+n^{\prime}$, and when $n^{\prime \prime} \geq n+n^{\prime}$, we have

$$
\begin{aligned}
Q_{n n^{\prime}}^{n^{\prime \prime}+1}(1,1,2)= & \frac{\left(\frac{1}{2}\left(n^{\prime \prime}+n+n^{\prime}\right)\right) !\left(\frac{1}{2}\left(n^{\prime \prime}-n-n^{\prime}\right)\right) !}{\left(\frac{1}{2}\left(n^{\prime \prime}+n-n^{\prime}\right)\right) !\left(\frac{1}{2}\left(n^{\prime \prime}+n^{\prime}-n\right)\right) !} \\
& \times\left(\frac{1}{2}\right)^{n+n^{\prime}+1} P_{\frac{n^{\prime \prime}-n-n^{\prime}}{2}}^{\left(n, n^{\prime}\right)}(0) P_{\frac{n^{\prime \prime}-n-n^{\prime}}{2}}^{\left(n^{\prime}, n\right)}(0),
\end{aligned}
$$

also see Note 2 at the end of Section 5.

3. From a result of Bailey [35] it also follows that $Q_{n+1, n^{\prime}}^{m-m^{\prime}}$ and $Q_{n+1, n^{\prime}+2}^{m-m^{\prime}}$ vanish when $\rho \geq 2$, but this is already clear from the fact that the $Z$ 's are supported by the unit disk. The result in Eq. (69) takes a more complicated form when $0<\rho<2$, see Section 6 for more details.

4. In Subsection 7.2, a detailed computation, based on Theorem 4.1, for the OTF corresponding to $P=Z_{0}^{0}$ is presented.

\section{Cosine representation of the radial polynomials}

We shall prove the following result.

Theorem 5.1. Let $m, n$ be integers $\geq 0$ with $n-m$ even and non-negative. Then

$$
R_{n}^{m}(\cos x)=\sum_{j=0}^{\lfloor n / 2\rfloor} a_{n-2 j} \cos (n-2 j) x,
$$

where for integer $k \geq 0$ with $n-k$ even and non-negative

$$
a_{k}=\varepsilon_{k} \frac{p ! q !}{s ! t !}\left(\frac{1}{2}\right)^{l}\left(P_{p}^{(\gamma, \delta)}(0)\right)^{2} .
$$

Here $\varepsilon_{0}=1, \varepsilon_{1}=\varepsilon_{2}=\ldots=2$ (Neumann's symbol), and

$$
\begin{aligned}
& p=\frac{n-l}{2}, q=\frac{n+l}{2}, \quad s=\frac{n-r}{2}, \\
& t=\frac{n+r}{2}, \gamma=\frac{l-r}{2}, \delta=\frac{l+r}{2},
\end{aligned}
$$

where

$$
l=\max (m, k), \quad r=\min (m, k) .
$$

Proof. We have from Eq. (7)

$$
R_{n}^{m}(\cos x)=(-1)^{\frac{n-m}{2}} \int_{0}^{\infty} J_{n+1}(u) J_{m}(u \cos x) d u .
$$

Next we note that

$$
R_{n}^{m}(\cos x)=(\cos x)^{m} P_{\frac{n-m}{2}}^{(0, m)}(\cos 2 x)
$$

has non-vanishing Fourier components $b_{k} e^{i k x}$ only for integer $k$ of the same parity as $m$. For such $k$ we shall show that

$$
\frac{1}{2 \pi} \int_{0}^{2 \pi} J_{m}(u \cos x) e^{i k x} d x=J_{\frac{m-k}{2}}\left(\frac{1}{2} u\right) J_{\frac{m+k}{2}}\left(\frac{1}{2} u\right) .
$$

Indeed, abbreviating "the coefficient of $e^{i m y}$ in" by $C_{m}$, we have by the generating function

$$
e^{i z \sin y}=\sum_{m=-\infty}^{\infty} J_{m}(z) e^{i m y}
$$

that

$$
\begin{aligned}
& \frac{1}{2 \pi} \int_{0}^{2 \pi} J_{m}(u \cos x) e^{i k x} d x \\
& =\frac{1}{2 \pi} C_{m}\left[\int_{0}^{2 \pi} e^{i u \cos x \sin y} e^{i k x} d x\right] \\
& =\frac{1}{2 \pi} C_{m}\left[\int_{0}^{2 \pi} e^{\frac{1}{2} i u \sin (x+y)} e^{-\frac{1}{2} i u \sin (x-y)} e^{i k x} d x\right] \\
& =\frac{1}{2 \pi} C_{m}\left[\sum_{m_{1}, m_{2}=-\infty}^{\infty} J_{m_{1}}\left(\frac{1}{2} u\right) J_{m_{2}}\left(\frac{1}{2} u\right)\right. \\
& =C_{m}\left[\sum_{0}^{2 \pi} e^{i m_{1}(x+y)-i m_{2}(x-y)+i k x} d x\right] \\
& {\left[\sum_{\frac{m-k}{2}}^{\infty} J_{m_{1}, m_{2}=-\infty,}\left(\frac{1}{2} u\right) J_{m_{2}}\left(\frac{1}{2} u\right) e^{i\left(m_{1}+m_{2}\right) y}\right.} \\
& \left.\frac{m_{2}-m_{1}=k}{2} u\right) J_{\frac{m+k}{2}}\left(\frac{1}{2} u\right) .
\end{aligned}
$$

Then we have at once from Eq.(77) that

$$
\begin{aligned}
& \frac{1}{2 \pi} \int_{0}^{2 \pi} R_{n}^{m}(\cos x) e^{i k x} d x \\
& =(-1)^{\frac{n-m}{2}} \int_{0}^{\infty} J_{n+1}(u) J_{\frac{m-k}{2}}\left(\frac{1}{2} u\right) J_{\frac{m+k}{2}}\left(\frac{1}{2} u\right) d u .
\end{aligned}
$$

When $k \geq 0$ and $m-k \geq 0$, we have then

$$
\frac{1}{2 \pi} \int_{0}^{2 \pi} R_{n}^{m}(\cos x) e^{i k x} d x=2(-1)^{\frac{n-m}{2}} Q_{\frac{m-k}{2}, \frac{m+k}{2}}^{n+1}(1,1,2),
$$

see Eqs. (71) and (72). For this case, the result follows from Eq. (72), the fact that $P_{p}^{(\delta, \gamma)}(0)=(-1)^{p} P_{p}^{(\gamma, \delta)}(0)$ and the fact 
that $a_{k}=a_{-k}$. When $k \geq 0$ and $m-k \leq 0$, the result follows in a similar manner, by using that $J_{\frac{m-k}{2}}\left(\frac{1}{2} u\right)=(-1)^{\frac{m-k}{2}} J_{\frac{k-m}{2}}\left(\frac{1}{2} u\right)$ and Eq. (72) together with some easy administration with signs.

\section{Notes.}

1. It is straightforward to generalize the result of Theorem 5.1 to the representation of $R_{n}^{m}(v \cos x)$ with $0 \leq v \leq 1$. Now $Q_{\frac{m-k}{2}, \frac{m+k}{2}}^{n+1}(v, v, 2)$ appears in Eq. (83) and $a_{k}$ in Eq. (74) becomes

$$
a_{k}(v)=\varepsilon_{k} \frac{p ! q !}{s ! t !}\left(\frac{1}{2} v\right)^{l} P_{p}^{(\gamma, \delta)}(x) P_{p}^{(\gamma, \delta)}(-x)
$$

where $x=\left(1-v^{2}\right)^{1 / 2}$. Also, see Section 6 and Eq. (20).

2. In using Theorem 5.1 it is convenient to note that

$$
P_{p}^{(\gamma, \delta)}(0)=\frac{1}{2^{p}} \sum_{j=0}^{p}\left(\begin{array}{c}
p+\gamma \\
j
\end{array}\right)\left(\begin{array}{c}
p+\delta \\
p-j
\end{array}\right)(-1)^{p-j}
$$

see [18], 22.3.1 on p. 775. Alternatively, $P_{p}^{(\gamma, \delta)}$ can be evaluated per Eq. (90) in terms of the hypergeometric function ${ }_{1} F_{2}$.

3. In Subsection 7.3, a table is presented in which the radial polynomials $R_{n}^{m}$, integer $n, m$ with $0 \leq n, m \leq 8$ and $n-m \geq 0$ and even, are given in polynomial and in the cosine representation of Theorem 5.1.

\section{Infinite integrals involving the product of three Bessel functions}

We consider in this section the integrals

$$
A_{\lambda \mu \nu}(a, b, c)=\int_{0}^{\infty} J_{\lambda}(a x) J_{\mu}(b x) J_{\nu}(c x) d x
$$

where $a, b, c>0$ and $\lambda, \mu, v$ are non-negative integers. There is quite some literature on these integrals and more general instances of them, see [36], Sec. 13.46 on pp. 411-415, [35], [37], Sec. 19.3 on pp. 349-357, [38], Sec. 13.4 .5 on pp. 331-335. These general results can become quite unmanageable; it is the point of this section that, in the special cases that we consider, often concise and manageable results appear, often in terms of the radial polynomials themselves.

For the case that $a+b<c$ (and $a>0, b>0, c>0$ ), the integral in Eq. (86) is given by Bailey, [35], Eq. (8.1) (with a minor correction in which the $c^{\mu+v+1}$ in the denominator at the right-hand side should be replaced by $c$ ), as

$$
\begin{aligned}
& \int_{0}^{\infty} J_{\lambda}(c u \sin \alpha \cos \beta) J_{\mu}(c u \cos \alpha \sin \beta) J_{v}(c u) d u \\
&= \frac{\Gamma\left(\frac{1}{2}(1+\lambda+\mu+v)\right) \sin ^{\lambda} \alpha \cos ^{\lambda} \beta \cos ^{\mu} \alpha \sin ^{\mu} \beta}{c \Gamma(\lambda+1) \Gamma(\mu+1) \Gamma\left(\frac{1}{2}(1-\lambda-\mu+v)\right)} \\
& \times{ }_{2} F_{1}\left(\frac{1}{2}(1+\lambda+\mu-v), \frac{1}{2}(1+\lambda+\mu+v) ;\right. \\
&\left.\lambda+1 ; \sin ^{2} \alpha\right) \\
& \times{ }_{2} F_{1}\left(\frac{1}{2}(1+\lambda+\mu-v), \frac{1}{2}(1+\lambda+\mu+v) ;\right. \\
&\left.\mu+1 ; \sin ^{2} \beta\right),
\end{aligned}
$$

where $\alpha, \beta \geq 0$ are such that $\alpha+\beta<\pi / 2$. In Eq. (20) the choice

$$
\begin{gathered}
c=1, \quad a=\sin \alpha \cos \beta, \quad b=\cos \alpha \sin \beta ; \\
\lambda=m-m^{\prime}, \quad \mu=n^{\prime}, \quad v=n+1
\end{gathered}
$$

is made. When $m-m^{\prime} \geq 0$ and $A=\sin \alpha, B=\sin \beta$, we have

$$
\begin{aligned}
& \int_{0}^{\infty} J_{m-m^{\prime}}(a u) J_{n^{\prime}}(b u) J_{n+1}(u) d u \\
&= \frac{\Gamma\left(\frac{1}{2}\left(n+n^{\prime}+m-m^{\prime}\right)+1\right) a^{m-m^{\prime}} b^{n^{\prime}}}{\Gamma\left(m-m^{\prime}+1\right) \Gamma\left(n^{\prime}+1\right) \Gamma\left(\frac{1}{2}\left(n-n^{\prime}-m+m^{\prime}\right)+1\right)} \\
& \times{ }_{1} F_{2}\left(\frac{1}{2}\left(n^{\prime}-n+m-m^{\prime}\right), \frac{1}{2}\left(n+n^{\prime}+m-m^{\prime}\right)+1 ;\right. \\
&\left.m-m^{\prime}+1 ; A^{2}\right) \\
& \times{ }_{1} F_{2}\left(\frac{1}{2}\left(n^{\prime}-n+m-m^{\prime}\right), \frac{1}{2}\left(n+n^{\prime}+m-m^{\prime}\right)+1 ;\right. \\
&\left.n^{\prime}+1 ; B^{2}\right) .
\end{aligned}
$$

Recall that both $n-m$ and $n^{\prime}-m^{\prime}$ are even. Hence $n-n-m^{\prime}+m^{\prime}$ is an even integer, and when this even integer is negative the whole expression (89) vanishes due to the $\Gamma\left(1 / 2\left(n-n^{\prime}-m+m^{\prime}\right)+1\right)$ in the denominator. Using [18], 15.4.6 on p. 561 and 22.4.1 on p. 777, we have for $j=0,1, \ldots$ and $\gamma, \delta \geq 0$

$$
\begin{aligned}
& { }_{1} F_{2}(-j, \gamma+1+\delta+j ; \gamma+1 ; x) \\
& =\frac{j ! \Gamma(\gamma+1)}{\Gamma(\gamma+1+j)} P_{j}^{(\gamma, \delta)}(1-2 x) .
\end{aligned}
$$


Therefore,

$$
\begin{aligned}
&{ }_{1} F_{2}\left(\frac{1}{2}\left(n^{\prime}-n+m-m^{\prime}\right), \frac{1}{2}\left(n+n^{\prime}+m-m^{\prime}\right)+1 ;\right. \\
&\left.m-m^{\prime}+1 ; A^{2}\right) \\
&={ }_{1} F_{2}\left(\frac{1}{2}\left(n^{\prime}-n+m-m^{\prime}\right), m-m^{\prime}+1+n^{\prime}\right. \\
&\left.\quad+\frac{1}{2}\left(n-n^{\prime}-m+m^{\prime}\right) ; m-m^{\prime}+1 ; A^{2}\right) \\
&=\frac{\left(\frac{1}{2}\left(n^{\prime}-n-m+m^{\prime}\right)\right) !\left(m-m^{\prime}\right) !}{\left(\frac{1}{2}\left(n-n^{\prime}+m-m^{\prime}\right)\right) !} \\
& \quad \times P_{\frac{1}{2}\left(n-m^{\prime}-n^{\prime}\right)}^{\left(m-m+m^{\prime}\right)}\left(1-2 A^{2}\right),
\end{aligned}
$$

and similarly

$$
\begin{aligned}
& { }_{1} F_{2}\left(\frac{1}{2}\left(n^{\prime}-n+m-m^{\prime}\right), \frac{1}{2}\left(n+n^{\prime}+m-m^{\prime}\right)+1 ; n^{\prime}\right. \\
& \left.\quad+1 ; B^{2}\right) \\
& =\frac{\left(\frac{1}{2}\left(n-n^{\prime}-m+m^{\prime}\right)\right) !\left(n^{\prime}\right) !}{\left(\frac{1}{2}\left(n+n^{\prime}-m+m^{\prime}\right)\right) !} P_{\frac{1}{2}\left(n-n^{\prime}-m+m^{\prime}\right)}^{\left(n^{\prime} m-m^{\prime}\right)}\left(1-2 B^{2}\right) .
\end{aligned}
$$

Using that $P_{j}^{(\gamma, \delta)}(-x)=(-1)^{j} P_{j}^{(\delta, \gamma)}(x)$ in Eq. (92) and some further administration with $\Gamma$-functions and factorials then yields Eq. (21). In the case that $m^{\prime}-m \geq 0$, we use that $J_{m-m^{\prime}}(z)=(-1)^{m-m^{\prime}} J_{m^{\prime}-m}(z)$, and so we can apply Eq. (21) with $m$ and $m^{\prime}$ interchanged. This requires a careful administration with $q, p, q^{\prime}, p^{\prime}$ as well as with the signs $(-1)^{p-p^{\prime}}$ in Eq. (20). Doing so, Eq. (22) follows.

The result in Eqs. (21)-(23) has been proved now for the case that $a+b<1$. However, the case that $a+b=1, a>0, b>0$, follows by taking the limit case in Eq. (20) and observing that the integral in Eq. (20) converges uniformly in $(a, b) \in[\varepsilon, 1] \times$ $[\varepsilon, 1]$ for any $\varepsilon>0$ since $J_{k}(z)=O\left(z^{-1 / 2}\right), z \rightarrow \infty$ and $J_{k}(z)$ is bounded in $z \geq 0$.

We shall now consider the $Q$-integrals in Eqs. (71) and Eqs. (82), (83). These can be treated in very much the same way as the integrals in Eq. (89) that arise from Eq. (87) by making the choice as in Eq. (88). Note that we have here the limit case $a=b=\frac{1}{2} c$. As to Eq. (72), we note that $Q_{n n^{\prime}}^{n^{\prime \prime}+1}(1,1,2)=\frac{1}{2} Q_{n n^{\prime}}^{n^{\prime \prime}+1}\left(\frac{1}{2}, \frac{1}{2}, 1\right)$. We thus need to replace $\left(m-m^{\prime}, n^{\prime}, n+1\right)$ by $\left(n, n^{\prime}, n^{\prime \prime}+1\right)$ and take

$$
a=b=\frac{1}{2}=A^{2}=B^{2}
$$

in Eq. (89). In particular, the $Q$-integral vanishes when $n^{\prime}+n-n^{\prime \prime}<0$, and when $n^{\prime}+n-n^{\prime \prime} \geq 0$ the ${ }_{1} F_{2}$ that arise in Eqs. (91), (92) should be written down with the replacement just mentioned and the choice in Eq. (93). This then yields Eq. (72). Next, the $Q$-integral in Eq. (82) can be handled in a similar fashion by replacing $\left(m-m^{\prime}, n^{\prime}, n+1\right)$ by $\left(\frac{1}{2}(m-k), \frac{1}{2}(m+k), n+1\right)$ with $a, b, A, B$ as in Eq. (93).

The result for $Z_{n}^{m} * *_{c o r r} Z_{n^{\prime}}^{m^{\prime}}$ in Eq.(69) involves the integrals $Q$ in Eq. (71) with values of the numbers $a, b, c$ such that none of them is larger or equal than the sum of the other two when $0<$ $\rho<2$. For such integrals, there is a result, see [36], Eq.(7) on p.413, in the form of an infinite sum involving the product of three hypergeometric functions ${ }_{2} F_{1}$. This formula takes a more tractable form, solely in terms of radial polynomials, when $i=0, j=m, k=n+1$ in Eq.(71) with $n-|m|$ even and non-negative, see [28], Section 6 for more details.

\section{Examples}

In this section we present worked out examples of our main results.

\subsection{Example for Section 3}

We use Theorem 3.1 for the computation of the Zernike expansion of the scaled-and-shifted circle polynomial $Z_{4}^{0}$. We have

$$
Z_{4}^{0}\left(a+b \rho^{\prime} e^{i \vartheta^{\prime}}\right)=\sum_{n^{\prime}, m^{\prime}} K_{4 n^{\prime}}^{0 m^{\prime}} Z_{n^{\prime}}^{m^{\prime}}\left(\rho^{\prime} e^{i \vartheta^{\prime}}\right)
$$

where we have $K_{4 n^{\prime}}^{0 m^{\prime}} Z_{n^{\prime}}^{m^{\prime}} \not \equiv 0$ only if $n^{\prime}$ and $m^{\prime}$ have the same parity and $\left|m^{\prime}\right| \leq n^{\prime} \leq 4-m^{\prime}$, see Eq. (50). This leaves us with the cases

$$
\begin{array}{cc}
\left|m^{\prime}\right|=0, \quad n^{\prime}=0,2,4 ; \quad & \left|m^{\prime}\right|=1, \quad n^{\prime}=1,3 ; \\
& \left|m^{\prime}\right|=2, \quad n^{\prime}=2 .
\end{array}
$$

Furthermore, $K_{4 n^{\prime}}^{0,-m^{\prime}}=K_{4 n^{\prime}}^{0 m^{\prime}}$ and so it is sufficient to do the computations for the cases $m^{\prime}=0,1,2$ in Eq. (95).

$\underline{m^{\prime}=0 \text {. a. }} K_{40}^{00}=T_{40}^{00}-T_{42}^{00}$ by Eq. (19) with $T_{40}^{00}$ and $T_{42}^{00}$ given by Eq. (22) as

$$
\begin{aligned}
& \quad T_{40}^{00}\left[p=q=2 ; p^{\prime}=q^{\prime}=0\right] \\
& =P_{2}^{(0,0)}\left(1-2 A^{2}\right) P_{2}^{(0,0)}\left(2 B^{2}-1\right), \\
& T_{42}^{00}\left[p=q=2 ; p^{\prime}=q^{\prime}=1\right] \\
& =b^{2} P_{1}^{(0,2)}\left(1-2 A^{2}\right) P_{1}^{(0,2)}\left(2 B^{2}-1\right) .
\end{aligned}
$$

$\underline{m^{\prime}=0 . \text { b. }} K_{42}^{00}=T_{42}^{00}-T_{44}^{00}$ with $T_{42}^{00}$ given in Eq. (97) and $T_{44}^{00}$ given as

$$
\begin{aligned}
& T_{44}^{00}\left(p=q=2 ; p^{\prime}=q^{\prime}=2\right] \\
& =b^{4} P_{0}^{(0,4)}\left(1-2 A^{2}\right) P_{0}^{(0,4)}\left(2 B^{2}-1\right) .
\end{aligned}
$$

$\frac{m^{\prime}=0 \text {. c. }}{T_{42}^{00}}=T_{44}^{00}-T_{46}^{00}$ with $T_{44}^{00}$ given in Eq. (98) and $T_{46}^{00}=0$.

$\underline{m^{\prime}=1 \text {. a. }} K_{41}^{01}=T_{41}^{01}-T_{43}^{01}$ by Eq. (19) with $T_{41}^{01}$ and $T_{43}^{01}$ given by Eq. (22) as

$$
\begin{aligned}
& T_{41}^{01}\left[p=q=2 ; p^{\prime}=0, q^{\prime}=1\right] \\
& =\frac{3}{2} a b P_{1}^{(1,1)}\left(1-2 A^{2}\right) P_{1}^{(1,1)}\left(2 B^{2}-1\right), \\
& T_{43}^{01}\left[p=q=2 ; p^{\prime}=1, q^{\prime}=2\right] \\
& =4 a b^{3} P_{0}^{(1,3)}\left(1-2 A^{2}\right) P_{0}^{(1,3)}\left(2 B^{2}-1\right) .
\end{aligned}
$$

$\underline{m^{\prime}=1 . b .} K_{43}^{01}=T_{43}^{01}-T_{45}^{01}$ with $T_{43}^{01}$ given by Eq. (100) and $T_{45}^{01}=0$. 
$\underline{m^{\prime}=2 \text {. a. }} K_{42}^{02}=T_{42}^{02}-T_{44}^{02}$ by Eq. (19) with $T_{42}^{02}$ given by Eq. (22) as

$$
\begin{aligned}
& T_{42}^{02}=\left[p=q=2 ; p^{\prime}=0, q^{\prime}=2\right] \\
& =6 a^{2} b^{2} P_{0}^{(2,2)}\left(1-2 A^{2}\right) P_{0}^{(2,2)}\left(2 B^{2}-1\right),
\end{aligned}
$$

and $T_{44}^{02}=0$ since $n-n^{\prime \prime}=0<2=\left|m-m^{\prime \prime}\right|$, see Eq. (23).

There remains to be calculated the right-hand side of Eqs. (96)-(101) with $P_{k}^{(\gamma, \delta)}$ the Jacobi polynomials and $1-2 A^{2}$ and $2 B^{2}-1$ given in terms of $a$ and $b$ by Eqs. (25), (26). In general, one can use that

$$
\begin{aligned}
P_{k}^{(\gamma, \delta)}(x)= & \frac{(k+\gamma) !}{k !(k+\gamma+\delta) !} \\
& \times \sum_{l=0}^{k}\left(\begin{array}{c}
k \\
l
\end{array}\right) \frac{(k+l+\gamma+\delta) !}{2^{l}(l+\gamma) !}(x-1)^{l}
\end{aligned}
$$

together with $P_{k}^{(\gamma, \delta)}(-x)=(-1)^{k} P_{k}^{(\delta, \gamma)}(x)$. For the present purposes it is sufficient to know that

$$
\begin{gathered}
P_{2}^{(0,0)}(x)=\frac{3}{2} x^{2}-\frac{1}{2} ; \quad P_{1}^{(0, \delta)}(x)=\left(1+\frac{1}{2} \delta\right) x-\frac{1}{2} \delta ; \\
P_{0}^{(\gamma, \delta)}(x)=1 .
\end{gathered}
$$

Using Eqs. (103) and (25), (26) in Eqs. (96)-(101) yields

$$
\begin{aligned}
& T_{40}^{00}=6 a^{4}+6 b^{4}+24 a^{2} b^{2}-6 a^{2}-6 b^{2}+1, \\
& T_{42}^{00}=b^{2}\left(12 a^{2}+4 b^{2}-3\right), \quad T_{44}^{00}=b^{4}, \\
& T_{41}^{01}=\frac{3}{2} a b\left(8 a^{2}+8 b^{4}-4\right), \quad T_{43}^{01}=4 a b^{3}, \\
& T_{42}^{02}=6 a^{2} b^{2} .
\end{aligned}
$$

This then gives

$$
\begin{aligned}
& K_{40}^{00}=6 a^{4}+2 b^{4}+12 a^{2} b^{2}-6 a^{2}-3 b^{2}+1, \\
& K_{42}^{00}=3 b^{4}+12 a^{2} b^{2}-3 b^{2}, \quad K_{44}^{00}=b^{4}, \\
& K_{41}^{01}=12 a^{3} b+8 a b^{3}-6 a b, \quad K_{43}^{01}=4 a b^{3}, \\
& K_{42}^{02}=6 a^{2} b^{2} .
\end{aligned}
$$

Hence

$$
\begin{aligned}
Z_{4}^{0}\left(a+b \rho^{\prime} e^{i \vartheta^{\prime}}\right) & \\
= & \left\{\left(6 a^{4}+2 b^{4}+12 a^{2} b^{2}-6 a^{2}-3 b^{2}+1\right) Z_{0}^{0}\right. \\
& \left.+\left(3 b^{4}+12 a^{2} b^{2}-3 b^{2}\right) Z_{2}^{0}+b^{4} Z_{4}^{0}\right\} \\
& +\left\{\left(12 a^{3}+8 a b^{3}-6 a b\right) Z_{1}^{1}\right. \\
& +\left(12 a^{3} b+8 a b^{3}-6 a b\right) Z_{1}^{-1} \\
& \left.+4 a b^{3} Z_{3}^{1}+4 a b^{3} Z_{3}^{-1}\right\} \\
& +\left\{6 a^{2} b^{2} Z_{2}^{2}+6 a^{2} b^{2} Z_{2}^{-2}\right\}
\end{aligned}
$$

where the $Z_{n^{\prime}}^{m^{\prime}}$ at the right-hand side of Eq. (110) should be evaluated at $\rho^{\prime} e^{i \vartheta^{\prime}}$.
It is obvious that for the expansion of a general $Z_{n}^{m}\left(a+b \rho^{\prime} e^{i \vartheta^{\prime}}\right)$ one can construct a concise and efficient computer code on basis of Theorem 3.1, taking advantage of the various shortcuts and reuse of intermediate results such as those encountered in passing in the above example.

\subsection{Example for Section 4}

We compute, using Theorem 4.1 and the notes thereafter, the Zernike expansion of $Z_{0}^{0} * *_{\text {corr }} Z_{0}^{0}$. It is easy to show by elementary means, $Z_{0}^{0} * *_{\text {corr }} Z_{0}^{0}$ being the area of the common part of two disks of radius 1 whose centers are at a distance $\rho$ apart, that

$$
\begin{gathered}
\left(Z_{0}^{0} * *_{\operatorname{corr}} Z_{0}^{0}\right)(\rho)=2\left[\arccos \left(\frac{1}{2} \rho\right)-\frac{1}{2} \rho \sqrt{1-\left(\frac{1}{2} \rho\right)^{2}}\right], \\
\text { where } 0 \leq \rho \leq 2 .
\end{gathered}
$$

From Eq. (60) we have

$$
\left(Z_{0}^{0} * *_{\operatorname{corr}} Z_{0}^{0}\right)(\rho)=2 \pi \int_{0}^{\infty} \frac{J_{1}^{2}(u) J_{0}(\rho u)}{u} d u
$$

This integral can be found in [39], 2.12.42, item 31 on p. 232, and this would yield Eq. (111) when the parentheses would have been placed correctly in this reference (a cross-check with [39], 2.12.42, item 15 on p. 230, that arises when the integral on the right-hand side of Eq. (112) is differentiated with respect to $\rho$, shows inconsistency of [39] in this matter).

We have $m^{\prime \prime}=m-m^{\prime}=0$ and $n^{\prime \prime}$ is even at the right-hand side of Eq. (61), and this yields

$$
\begin{gathered}
\left(Z_{0}^{0} * *_{\text {corr }} Z_{0}^{0}\right)(\rho)=\frac{\pi}{4} \sum_{n^{\prime \prime} \text { even }, \geq 0}\left(n^{\prime \prime}+1\right) \Gamma_{00 n^{\prime \prime}}^{000} Z_{n^{\prime \prime}}^{0}\left(\frac{1}{2} \rho\right), \\
\text { where } 0 \leq \rho \leq 2,
\end{gathered}
$$

where

$$
\begin{aligned}
\Gamma_{00 n^{\prime \prime}}^{000} & =8(-1)^{-\frac{1}{2} n^{\prime \prime}} \int_{0}^{\infty} J_{1}(u) J_{1}(u) J_{n^{\prime \prime}+1}(2 u) \frac{d u}{u^{2}} \\
& =2(-1)^{\frac{1}{2} n^{\prime \prime}}\left[Q_{00}^{n^{\prime \prime}+1}+2 Q_{02}^{n^{\prime \prime}+1}+Q_{22}^{n^{\prime \prime}+1}\right]
\end{aligned}
$$

with, see Eq.(72),

$$
\begin{aligned}
Q_{00}^{n^{\prime \prime}+1}= & \frac{1}{2}\left(P_{\frac{1}{2} n^{\prime \prime}}^{(0,0)}(0)\right)^{2}, \\
& \text { where } n^{\prime \prime}=0,2, \ldots, \\
Q_{02}^{n^{\prime \prime}+1}= & \frac{1}{8} P_{\frac{1}{2} n^{\prime \prime}-1}^{(0,2)}(0) P_{\frac{1}{2} n^{\prime \prime}-1}^{(2,0)}(0), \\
& \text { where } n^{\prime \prime}=2,4, \ldots, \\
Q_{22}^{n^{\prime \prime}+1}= & \frac{\left(\frac{1}{2} n^{\prime \prime}+2\right) !\left(\frac{1}{2} n^{\prime \prime}-2\right) !}{\left(\left(\frac{1}{2} n^{\prime \prime}\right) !\right)^{2}} \frac{1}{32} \\
& \times\left(P_{\frac{1}{2} n^{\prime \prime}-2}^{(2,2)}(0)\right)^{2}, \\
& \text { where } n^{\prime \prime}=4,6, \ldots,
\end{aligned}
$$


while $Q_{02}^{1}$ and $Q_{22}^{1}, Q_{22}^{3}$ vanish. Thus this yields the Zernike ${ }^{0}$-expansion of the function

$$
2\left(\arccos \tau-\tau \sqrt{1-\tau^{2}}\right)=\sum_{n^{\prime \prime} \text { even }, \geq 0} C_{n^{\prime \prime}}^{0} Z_{n^{\prime \prime}}^{0}\left(\tau=\frac{1}{2} \rho\right),
$$

where $0 \leq \tau \leq 1$

The $C_{n^{\prime \prime}}^{0}$ are given in integral form as

$$
\begin{aligned}
C_{n^{\prime \prime}}^{0}= & 4\left(n^{\prime \prime}+1\right), \\
& \times \int_{0}^{1}\left(\arccos \tau-\tau \sqrt{1-\tau^{2}}\right) R_{n^{\prime \prime}}^{0}(\tau) \tau d \tau ;
\end{aligned}
$$

the evaluation of the integrals in Eq. (119) becomes cumbersome, already for low values of $n^{\prime \prime}=0,2, \ldots$. We compute from Eqs. (114)-(118) and $C_{n^{\prime \prime}}^{0}=\frac{\pi}{4}\left(n^{\prime \prime}+1\right) \Gamma_{00 n^{\prime \prime}}^{000}$

$$
C_{0}^{0}=\frac{\pi}{4}, C_{2}^{0}=-\frac{3 \pi}{8}, C_{4}^{0}=\frac{5 \pi}{32}, \ldots
$$

Unfortunately, see Eq. (85), there does not seem to exist a closed formula for the values of $P_{k}^{(\gamma, \delta)}(0)$ as required in Eqs. (115)-(117), except for the case $\gamma=\delta=0$, see [18], Table 22.4.1 on p. 777. Furthermore, the $C_{\eta^{\prime \prime}}^{0}$ decay only slowly because of non-smooth behaviour of $\left(Z_{0}^{0} * *_{\text {corr }} Z_{0}^{0}\right)(v, \mu)$ around $(v, \mu)=(0,0)$ and, to a lesser extent, around $v^{2}+\mu^{2}=4$, where $\left(Z_{0}^{0} * *_{\text {corr }} Z_{0}^{0}\right)(\rho)$ is continuous differentiable, but not twice differentiable with respect to $\rho$.

\subsection{Examples for Section 5}

We have computed, using Theorem 5.1, the Fourier coefficients $a_{k}$ in the cosine representation

$$
R_{n}^{m}(\cos x)=\sum_{j=0}^{\lfloor n / 2\rfloor} a_{n-2 j} \cos (n-2 j) x
$$

for various cases of integer $n, m$ with $n, m$ non-negative and $n-m$ even and non-negative. The results are collected in Table 1 .
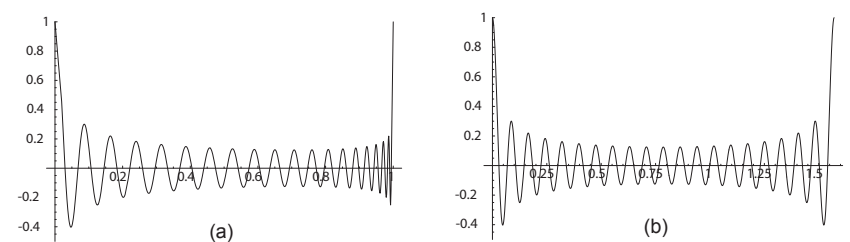

FIG. 5 Plot of (a) $R_{80}^{0}(\rho), 0 \leq \rho \leq 1$, and (b) $R_{80}^{0}(\cos x), 0 \leq x \leq \pi / 2$. The sampling rate used to display (a) is not high enough to adequately represent the last peak but one just before $\rho=1$.

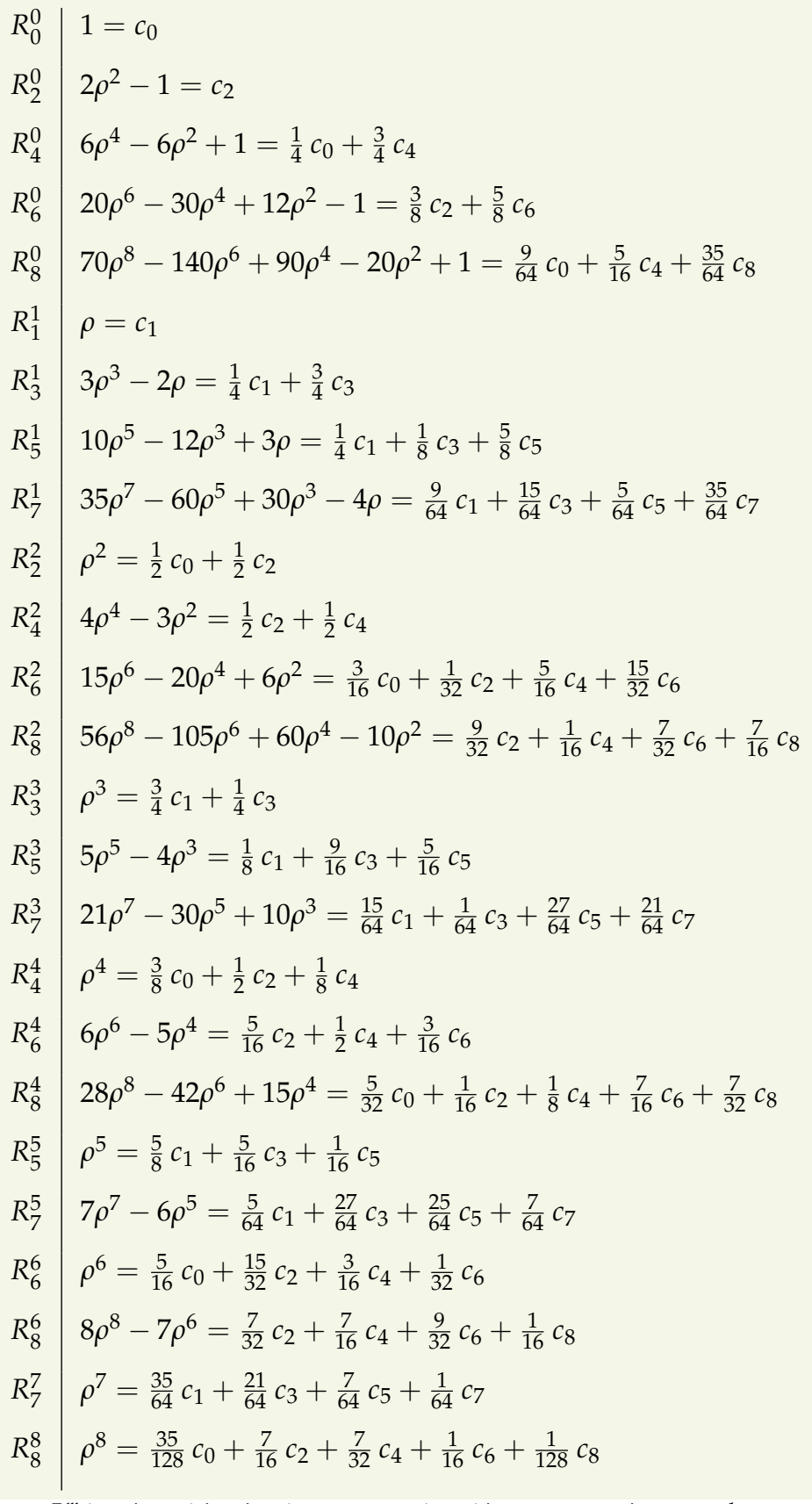

TABLE $1 R_{n}^{m}$ in polynomial and cosine representation with $\rho=\cos x$ and $c_{k}=\cos k x$

In Figure 5 we have displayed $R_{80}^{0}(\rho), 0 \leq \rho \leq 1$, and $R_{80}^{0}(\cos x), 0 \leq x \leq \pi / 2$ to illustrate the point that the variation of the radial polynomial is more or less spread out uniformly over the $x$-interval.

\section{Conclusion and outlook}

In this paper we have used the basic identity in the classical Nijboer-Zernike theory of optical aberrations to prove three new analytic results for the Zernike circle polynomials, and we have indicated the relevance of these new results for the fields of optical lithography, ophthalmology and computational optics. The first result is an analytic formula for the expansion coefficients of a circle polynomial $Z_{n}^{m}$ when it is considered on an arbitrary disk, contained in the nominal disk $\rho \leq 1$, and expanded in terms of the orthogonal circle polynomials pertaining to this smaller disk. This result embod- 
ies an analytic solution of the much studied problem in lithography and ophthalmology of how the expansion coefficients of a non-uniform complex pupil function change when this function is considered on sub-disks of the nominal disk. It would be interesting to see how this result generalizes when restriction to subdomains with elliptic, rather than circular, boundaries were considered. At present, there are no results for this more general situation, since the very choice of orthogonal polynomials on these more general subdomains is a non-trivial issue. The second result concerns the computation of the Zernike expansion of the optical transfer function (OTF) from the Zernike expansion of the complex pupil function that yields the OTF in the form of an autocorrelation function. This problem has received attention from researchers in the optics community for more than half a century, but a complete analytic solution was not presented yet. It is expected that the analytic solution presented in this paper will contribute, for instance, to faster calculation schemes for pattern transfer in high-resolution optical lithography and to faster operation of pupil function retrieval schemes in optics. The third result concerns the analytic determination of the Fourier coefficients of the radial polynomials $R_{n}^{|m|}(\rho)$ when the radial variable $\rho$, $0 \leq \rho \leq 1$, is replaced by $\cos x, 0 \leq x \leq \pi / 2$. The explicit form of these coefficients presented in this paper shows that they are all non-negative and small. Thus, this result offers, in conjunction with FFT-methods, an attractive prospect for methods to perform the radial integration in diffraction integrals.

The analytic formulas given in this paper for the quantities just mentioned contain factorials, monomials and Jacobi polynomials only, and are thus readily and numerically efficiently implemented. For all these three results, the quantities of interest have first been expressed, using the basic result of the Nijboer-Zernike theory, as integrals involving the product of three Bessel functions. In all cases, it appeared that the expressions for these integrals, as given in existing literature, can be reduced to the simpler form described above, making the application of the results of this paper quite feasible.

\section{A Convergence of the integral in}

\section{Eq. (7)}

We shall show in this appendix that for non-negative integers $n$ and $m$ with $n-m$ even and non-negative, the integral

$$
\int_{0}^{v} J_{n+1}(u) J_{m}(\rho u) d u
$$

as $v \rightarrow \infty$ converges to $(-1)^{\frac{n-m}{2}} R_{n}^{m}(\rho)$ for $0 \leq \rho<1$ and to 0 for $\rho>1$, and that it does so boundedly in $\rho \geq 0$ and uniformly in $\rho$ outside $(1-\varepsilon, 1+\varepsilon)$ for any $\varepsilon>0$. We have from [18], 9.2.1 on p. 364

$$
\begin{gathered}
J_{k}(u)=\sqrt{\frac{2}{\pi u}} \cos \left(u-\frac{1}{2} k \pi-\frac{1}{4} \pi\right)+O\left(u^{-3 / 2}\right), \\
\text { where } u \rightarrow \infty,
\end{gathered}
$$

and $J_{k}(u)$ is smooth and bounded on $u \geq 0$. Therefore, to show bounded and uniform convergence of the integral in
Eq. (122) as $v \rightarrow \infty$ on the appropriate sets of $\rho$, it is sufficient to establish this for the integral

$$
\begin{aligned}
\frac{2}{\pi} \int_{0}^{v}[ & \frac{1}{u} \cos \left(u-\frac{1}{2}(n+1) \pi-\frac{1}{4} \pi\right) \\
& \left.\times \cos \left(\rho u-\frac{1}{2} m \pi-\frac{1}{4} \pi\right)\right] d u .
\end{aligned}
$$

Once this has been established, the issue of to what the integral in Eq. (122) converges is settled by the remark that $Z_{n}^{m}$ and $2 \pi i^{n} \exp (i m \varphi) J_{n+1}(2 \pi r) / 2 \pi r$, see Eq. (5), are $2 D$ Fourier pairs so that Fourier inversion of the latter function yields the former in $L^{2}(\mathbb{R})$-sense while the former function is smooth outside the set $v^{2}+\mu^{2}=1$.

Using elementary trigonometric identities, we have

$$
\begin{aligned}
\frac{2}{\pi} \int_{1}^{v} \frac{1}{u} \cos \left(u-\frac{1}{2}(n+1) \pi-\frac{1}{4} \pi\right) \\
\quad \times \cos \left(\rho u-\frac{1}{2} m \pi-\frac{1}{4} \pi\right) d u \\
=\frac{(-1)^{p}}{\pi} \int_{1}^{v} \frac{\sin (1-\rho) u}{u} d u \\
-\frac{(-1)^{q}}{\pi} \int_{1}^{v} \frac{\cos (1+\rho) u}{u} d u \\
=\frac{(-1)^{p}}{\pi} \int_{1-\rho}^{(1-\rho) v} \frac{\sin x}{x} d x \\
\quad-\frac{(-1)^{q}}{\pi} \int_{1+\rho}^{(1+\rho) v} \frac{\cos x}{x} d x,
\end{aligned}
$$

where we have set $p=\frac{1}{2}(n-m), q=\frac{1}{2}(n+m)$. Since both functions

$$
\int_{0}^{y} \frac{\sin x}{x} d x, y \geq 0 ; \quad \int_{1}^{y} \frac{\cos x}{x} d x, y \geq 1,
$$

are bounded and have a finite limit as $y \rightarrow \infty$, the convergence of the integral in Eq. (124) is bounded in $\rho \geq 0$ and uniform in any closed set of $\rho^{\prime}$ s not containing 1 . The assumption that $n$ and $m$ have same parity is essential: $\int_{1}^{\infty} \frac{1}{u} \cos ^{2} u d u=\infty$.

\section{Acknowledgements.}

The author wishes to express his thanks to Prof. Joseph Braat for his continuous interest and encouragement when this research was carried out, to Prof. Erik Koelink for calling the reference [33] to the author's attention and for bringing the result of [33] in appropriate context. This research was supported by Agentschap NL through the KWR-project Metrology.

\section{References}

[1] M. Born, and E. Wolf, Principles of Optics Ch. 9 (Cambridge University Press, Cambridge, United Kingdom, 1999). 
[2] W. J. Tango, "The circle polynomials of Zernike and their application in optics" Appl. Phys. 13, 327-332 (1977).

[3] C. -J. Kim and R. R. Shannon, "Catalog of Zernike polynomials" in Applied Optics and Optical Engineering, R. R. Shannon, and J. C. Wyant, eds. 10, 193-221 (Academic Press, New York, 1987).

[4] J. J. M. Braat, S. van Haver, A. J. E. M. Janssen, and P. Dirksen, "Assessment of optical systems by means of point-spread functions", Progress in Optics E. Wolf, ed. 51, 349-468 (Elsevier, Amsterdam, The Netherlands, 2008).

[5] R. J. Noll, "Zernike polynomials and atmospheric turbulence" J. Opt. Soc. Am. 66, 207-211 (1976).

[6] V. N. Mahajan, "Zernike annular polynomials and optical aberrations of systems with annular pupils" Appl. Opt. 33, 8125-8127 (1994).

[7] S. Bará, J. Arines, J. Ares, and P. Prado, "Direct transformation of Zernike eye aberration coefficients between scaled, rotated, and/or displaced pupils" J. Opt. Soc. Am. A23, 2061-2066 (2006).

[8] G. -M. Dai, "Scaling Zernike expansion coefficients to smaller pupil sizes: a simpler formula" J. Opt. Soc. Am. A23, 539-543 (2006).

[9] R. M. Aarts, and A. J. E. M. Janssen, "On-axis and far-field sound radiation from resilient flat and dome-shaped radiators" J. Acoust. Soc. Am. 125, 1444-1455 (2009).

[10] R. M. Aarts, and A. J. E. M. Janssen, "Sound radiation quantities arising from a resilient circular radiator" J. Acoust. Soc. Am. 126, 1776-1787 (2009).

[11] R. M. Aarts, and A. J. E. M. Janssen, "Sound radiation from a resilient cap on a rigid sphere" J. Acoust. Soc. Am. 127, 2262-2273 (2010).

[12] F. Zernike, "Diffraction theory of the knife-edge test and its improved version, the phase-contrast method" Physica 1, 689-704 (1934).

[13] B. R. A. Nijboer, The Diffraction Theory of Aberrations, (Ph.D. thesis, University of Groningen, The Netherlands, 1942).

[14] S. van Haver, J. J. M. Braat, P. Dirksen, and A. J. E. M. Janssen, "High-NA aberration retrieval with the Extended Nijboer-Zernike vector diffraction theory" J. Europ. 0pt. Soc. Rap. Public. 1, 06004 (2006).

[15] J. J. M. Braat, P. Dirksen, A. J. E. M. Janssen, and S. van Haver, "Strehl intensity and optimum focus of high-numerical-aperture beams" J. Europ. Opt. Soc. Rap. Public. 2, 07008 (2007).

[16] J. J. M. Braat, S. van Haver, A. J. E. M. Janssen, and P. Dirksen, "Energy and momentum flux in a high-numerical-aperture beam using the extended Nijboer-Zernike diffraction formalism" J. Europ. Opt. Soc. Rap. Public. 2, 07032 (2007).

[17] J. J. M. Braat, S. van Haver, A. J. E. M. Janssen, and S. F. Pereira, "Image formation in a multilayer using the Extended NijboerZernike theory" J. Europ. Opt. Soc. Rap. Public. 4, 09048 (2009).

[18] M. Abramowitz and I. A. Stegun, Handbook of Mathematical Functions, (Dover Publications, New York, 1972).

[19] A. J. E. M. Janssen, S. van Haver, P. Dirksen, and J. J. M. Braat, "Zernike representation and Strehl ratio of optical systems with variable numerical aperture" J. Mod. Optic. 55, 1127-1157 (2008).

[20] S. A. Comastri, L. I. Perez, G. D. Pérez, G. Martin, and K. Bastida, "Zernike expansion coefficients: rescaling and decentring for dif- ferent pupils and evaluation of corneal aberrations" J. Opt. A: Pure Appl. 0p. 9, 209-221 (2007).

[21] A. J. E. M. Janssen, and P. Dirksen, “Computing Zernike polynomials of arbitrary degree using the discrete Fourier transform" J. Europ. Opt. Soc. Rap. Public. 2, 07012 (2007).

[22] A. J. E. M. Janssen, and P. Dirksen, "Concise formula for the Zernike coefficients of scaled pupils" J. Microlith. Microfab. 5, 030501 (2006).

[23] C. J. R. Sheppard, S. Campbell, and M. D. Hirschhorn, "Zernike expansion of separable functions of Cartesian coordinates" Appl. Optics 43, 3963-3966 (2004).

[24] A. M. Cormack, "Representation of a function by its line integrals, with some radiological applications, II" J. Appl. Phys. 35, 2908-2913 (1964).

[25] E. C. Kintner, and R. M. Sillitto, "A new "analytic" method for computing the optical transfer function" Opt. Acta 23, 607-619 (1976).

[26] E. C. Kintner, "An analytic recurrence for computing the crossmultiplication coefficients in an analytic OTF method" Opt. Acta 24, 1237-1246 (1977).

[27] R. M. Aarts, and A. J. E. M. Janssen, Acoustic holography for piston sound radiation with non-uniform velocity profiles, ( $17^{\text {th }}$ International Congress on Sound at Vibration, 1-6, Cairo, 18-22 July, 2010).

[28] A. J. E. M. Janssen, "Zernike circle polynomials and infinite integrals involving the product of Bessel functions" arXiv:1007.0667V1, 5 July, 2010; also available from Library of Eindhoven University of Technology ISBN: 978-90-386-2290-3.

[29] H. H. Hopkins, "The numerical evaluation of the frequency response of optical systems" P. Phys. Soc. Lond. B 70, 1002-1005 (1957).

[30] J. Macdonald, "The calculation of the optical transfer function" Opt. Acta 18, 269-290 (1971).

[31] 0. T. A. Janssen, S. van Haver, A. J. E. M. Janssen, H. P. Urbach and S. F. Pereira, "Extended Nijboer-Zernike (ENZ) based mask imaging: Efficient coupling of electro-magnetic field solvers and the ENZ imaging algorithm" Proc. SPIE 6924, 692410 (2008).

[32] G. Szegö, Orthogonal Polynomials (AMS, Providence, 1939, $4^{\text {th }}$ ed., 1975).

[33] T. Koornwinder, "Positivity proofs for linearization and connection coefficients of orthogonal polynomials satisfying an addition formula" J. Lond. Math. Soc. (2) 18, 101-114 (1978).

[34] E. Koelink, private communication, 2002.

[35] W. N. Bailey, "Some infinite integrals involving Bessel functions" Proc. Lond. Math. Soc. (2) 40, 37-48 (1936).

[36] G. N. Watson, A Treatise on the Theory of Bessel Functions (Cambridge University Press, Cambridge, United Kingdom, 1944).

[37] A. Erdélyi et al., Tables of integral transforms, Vol. 2 (McGraw-Hill, New York, 1954).

[38] Y. L. Luke, Integrals of Bessel functions (McGraw-Hill, New York, 1962).

[39] A. P. Prudnikov, Yu. A. Brychkov, and 0.I. Marichev, Integrals and Series, Volume 2: Special Functions (Gordon and Breach Science, New York, 1986). 